\title{
Highest Weight Representations of the Neveu-Schwarz and Ramond Algebras
}

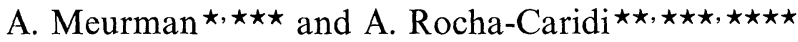 \\ Mathematical Sciences Research Institute, Berkeley, CA 94720, USA
}

\begin{abstract}
We construct a family of representations $\mathscr{K}^{\xi, w}$ of the NeveuSchwarz and Ramond algebras, which generalize the Fock representations of the Virasoro algebra. We show that the representations $\mathscr{K}^{\xi \text {,w }}$ are intertwined by a vertex operator.

The above results are used to give the proof of the conjectured formulas for the determinant of the contravariant form on the highest weight representations of the Neveu-Schwarz and Ramond algebras. Further results on the representation theory of the latter are derived from the determinant formulas.
\end{abstract}

\section{Introduction}

In Superstring theory physicists consider two supersymmetric extensions of the Lie algebra of vector fields on the circle $\left(\operatorname{Vect}\left(S^{1}\right)\right)$ called the Neveu-Schwarz [19] and Ramond [20] algebras. The Neveu-Schwarz algebra has basis $\left\{L_{0}^{\prime}, L_{i}, G_{j}\right\}$ $\left(i \in \mathbb{Z}, j \in \frac{1}{2}+\mathbb{Z}\right)$, where $L_{0}^{\prime}$ is central, and the bracket of two noncentral generators is given by the relations

$$
\begin{aligned}
& {\left[L_{i}, L_{j}\right]=(j-i) L_{i+j}+\delta_{i,-j}\left(\frac{i^{3}-i}{8}\right) L_{0}^{\prime},} \\
& {\left[G_{i}, G_{j}\right]=-2 L_{i+j}+\delta_{i,-j} \frac{1}{2}\left(i^{2}-\frac{1}{4}\right) L_{0}^{\prime},} \\
& {\left[L_{i}, G_{j}\right]=\left(j-\frac{1}{2} i\right) G_{i+j} .}
\end{aligned}
$$

The Ramond algebra has the same relations, but the $G_{j}$ are indexed by $\mathbb{Z}$. These algebras are " $\mathbb{Z}_{2}$-graded Lie algebras," i.e., $\mathbb{Z}_{2}$-graded vector spaces with a grading

* Current address: Department of Mathematics, University of Stockholm, Stockholm, Sweden $\star \star$ Current address: Department of Mathematics, Baruch College, Cuny, New York, NY 10010, USA

$\star \star \star$ Partially supported by the National Science Foundation through the Mathematical Sciences Research Institute

$\star \star \star \star$ Partially supported by NSF grant MCS- 8201260 
preserving bracket operation that satisfies $\mathbb{Z}_{2}$-graded versions of anticommutativity and Jacobi's identity.

In $[13,14] \mathrm{Kac}$ gave a formula for the determinant of the Shapovalov form for (a central extension of) Vect $\left(S^{1}\right)$. Kac's formula was later proved by Feigin and Fuchs $([2])^{1}$. In [14] Kac also gave a brief introduction to the highest weight theory of the Neveu-Schwarz algebra, and stated (without proof) a formula for its Shapovalov determinant. An interesting application of this formula is found in the recent work of Friedan et al. ([8]) where the formula is used to obtain a series of (conjectured) unitary representations. In [8] the authors also considered the Ramond algebra, for which they conjectured a determinant formula. In the present paper we prove the determinant formulas for both the Neveu-Schwarz and Ramond algebras $^{2}$ (Sect. 4). We then use the determinant formulas to prove that the highest weights of a Verma module and that of an irreducible subquotient are "linked" (Theorem 5.1). For the Neveu-Schwarz algebra this result was first stated by Kac [14].

Our proof of the determinant formulas follows the general lines of that of Feigin and Fuchs for Vect $\left(S^{1}\right)$ and is, like theirs, based on the construction of a concrete family of representations. Rather than generalizing the construction of [2], however, we generalize the one obtained by Goodman and Wallach [11]. One can show that these constructions are isomorphic via the boson-fermion correspondence [see Frenkel, I.B.: J. Funct. Anal. 44, 259-327 (1981)]. In this approach, the operator that was used in [2] to obtain highest weight vectors becomes a vertex operator.

We would like to point out that the theory of Verma modules for the Ramond algebra, developed in this paper, is quite a bit different from that of the NeveuSchwarz algebra. For example, the Ramond algebra admits "half" Verma modules as well as Verma modules. The former explain the extra factor in the determinant formula of this algebra.

Finally, we hope that our findings on the Ramond algebra, as well as our detailed follow-up and proofs of the results announced in [14] can serve as a basis for further investigations on the subject.

In Sect. 2 we review the background material on $\mathbb{Z}_{2}$-graded Lie algebras and their representations that will be needed in the paper. In Sect. 3 we present some basic results on the Neveu-Schwarz and Ramond algebras and their highest weight representations. The determinant formulas are stated at the end of this section. In Sect. 4 we construct the representations $\mathscr{K}^{\xi, w}$ (Theorem 4.2 ) and prove that they are intertwined by a vertex operator (Theorem 4.4). We then use the latter to obtain maximal weight vectors in $\mathscr{K}^{\xi, w}$ (Corollary 4.8 ). We conclude this section with the proof of the determinant formulas which consists of two parts: The degree computation (Proposition 4.10) and the proof of the vanishing of the determinant on the variety of its irreducible factors (Proposition 4.13). In Sect. 5 we construct Jantzen type filtrations following the general lines of [23], and then use them to obtain character sum formulas (Proposition 5.2). The latter imply Theorem 5.1

\footnotetext{
${ }^{1}$ After this work was completed we received reference [27]

${ }^{2}$ After this work was completed, D. Friedan informed us that Curtright and Thorn had also succeeded to prove these formulas
} 
mentioned above. Finally, in the Appendix, we extend to $\mathbb{Z}_{2}$-graded Lie algebras, some definitions and classic results about central extensions of Lie algebras.

We denote by $\mathbb{N}$ the set of positive integers, $\mathbb{Z}_{+}$the set of nonnegative integers, and set $\mathbb{Z}_{2}=\mathbb{Z} / 2 \mathbb{Z}$.

\section{Preliminaries}

First we recall the necessary definitions pertaining to $\mathbb{Z}_{2}$-graded algebras $([1,15,16,18])$. All algebras and vector spaces are defined over $\mathbb{C}$.

A $\mathbb{Z}_{2}$-graded algebra is an algebra $\mathfrak{a}$ with a vector space decomposition $\mathfrak{a}=\mathfrak{a}_{\overline{0}}+\mathfrak{a}_{\overline{1}}$ such that $\mathfrak{a}_{\alpha} \cdot \mathfrak{a}_{\beta} \subset \mathfrak{a}_{\alpha+\beta}$ for all $\alpha, \beta \in \mathbb{Z}_{2}$. The elements of $\mathfrak{a}_{\overline{0}}$ are called even and those of $a_{1}$ are called odd. By a subalgebra of a $\mathbb{Z}_{2}$-graded algebra we will always mean a $\mathbb{Z}_{2}$-graded subalgebra. Also, by a homomorphism $\Phi: \mathfrak{a} \rightarrow \mathfrak{a}^{\prime}$ of $\mathbb{Z}_{2}$-graded algebras we will always mean one that preserves the $\mathbb{Z}_{2}$-grading, i.e., such that $\Phi\left(\mathfrak{a}_{\alpha}\right) \subset \mathfrak{a}_{\alpha}^{\prime}$ for all $\alpha \in \mathbb{Z}_{2}$.

A $\mathbb{Z}_{2}$-graded Lie algebra is a $\mathbb{Z}_{2}$-graded vector space $\mathfrak{I}=\mathfrak{I}_{\overline{0}} \oplus \mathfrak{I}_{\overline{1}}$ equipped with a bilinear map $[$,$] such that \left[\mathfrak{l}_{\alpha}, \mathfrak{l}_{\beta}\right] \subset \mathfrak{l}_{\alpha+\beta}, \alpha, \beta \in \mathbb{Z}_{2}$, and satisfying the following conditions:

(L1) $[X, Y]=-(-1)^{\alpha \beta}[Y, X], X \in \mathfrak{l}_{\alpha}, Y \in \mathfrak{I}_{\beta}, \alpha, \beta \in \mathbb{Z}_{2}$,

(L2) $(-1)^{\alpha \gamma}[X,[Y, Z]]+(-1)^{\beta \alpha}[Y,[Z, X]]+(-1)^{\gamma \beta}[X,[Z, Y]]=0$,

$X \in \mathfrak{I}_{\alpha}, Y \in \mathfrak{I}_{\beta}, Z \in \mathbb{I}_{\gamma}, \alpha, \beta, \gamma \in \mathbb{Z}_{2}$.

The subalgebra $\mathfrak{I}_{\overline{0}}$ of $\mathfrak{I}$ is clearly a Lie algebra in the usual sense.

Note. We warn the reader that the terminology for the objects just defined is varied. $[15,16]$ use the term "Lie superalgebras." In [18] the authors consider what we would call " $\mathbb{Z}_{+}$-graded Lie algebras" and refer to them simply as "Lie algebras."

If $\mathfrak{a}$ is an associative $\mathbb{Z}_{2}$-graded algebra, set $[X, Y]=X Y-(-1)^{\alpha \beta} Y X, X \in \mathfrak{a}_{\alpha}$, $Y \in \mathfrak{a}_{\beta}, \alpha, \beta \in \mathbb{Z}_{2}$. We denote by $\mathfrak{a}_{L}$ the resulting $\mathbb{Z}_{2}$-graded Lie algebra.

The universal enveloping algebra of a $\mathbb{Z}_{2}$-graded Lie algebra $\mathfrak{I}$ is a pair $(U(\mathrm{l}), \sigma)$ where $U(\mathfrak{l})$ is an associative $\mathbb{Z}_{2}$-graded algebra and $\sigma: \mathfrak{l} \rightarrow U(\mathfrak{l})$ is a linear map preserving the $\mathbb{Z}_{2}$-grading, with the following universal property: $\sigma[X, Y]$ $=\sigma(X) \sigma(Y)-(-1)^{\alpha \beta} \sigma(Y) \sigma(X)$ for all $X \in \mathfrak{I}_{\alpha}, \quad Y \in \mathfrak{I}_{\beta}, \alpha, \beta \in \mathbb{Z}_{2}$, and given an associative $\mathbb{Z}_{2}$-graded algebra $\mathfrak{a}$ and a linear map $\tau: \mathfrak{l} \rightarrow \mathfrak{a}$ preserving the $\mathbb{Z}_{2}$-grading, and such that $\tau[X, Y]=\tau(X) \tau(Y)-(-1)^{\alpha \beta} \tau(Y) \tau(X)$ for $X \in \mathfrak{l}_{\alpha}, Y \in \mathfrak{l}_{\beta}$, $\alpha, \beta \in \mathbb{Z}_{2}$, then there is a unique homomorphism $\psi: U(\mathfrak{l}) \rightarrow \mathfrak{a}$ such that $\tau=\psi \circ \sigma$.

Let $V=V_{\overline{0}} \oplus V_{\overline{1}}$ be a $\mathbb{Z}_{2}$-graded vector space. Let, for $p \geqq 1,\left(\otimes^{p} V\right)_{\alpha}$ $=\sum_{\beta_{1}+\ldots+\beta_{p}=\alpha} V_{\beta_{1}} \otimes \ldots \otimes V_{\beta_{p}}, \alpha, \beta_{i} \in \mathbb{Z}_{2}, i=1, \ldots, p$, and set $T^{p}(V)=\otimes{ }^{p} V, p \geqq 1$, $T^{0}(V)=\mathbb{C}$. The tensor algebra $T(V)=\bigoplus_{p=0}^{\infty} T^{p}(V)$ is clearly a $\mathbb{Z}_{2}$-graded associative
algebra.

Concretely, $U(\mathfrak{l})=T(\mathfrak{l}) / I$, where $I$ is the two-sided $\left(\mathbb{Z}_{2}\right.$-graded) ideal in $T(\mathfrak{l})$ generated by the elements of the form $X \otimes Y-(-1)^{\alpha \beta} Y \otimes X-[X, Y], X \in \mathfrak{l}_{\alpha}, Y \in \mathfrak{l}_{\beta}$, $\alpha, \beta \in \mathbb{Z}_{2}$, and $\sigma: \mathfrak{I} \rightarrow U(\mathfrak{l})$ is induced by the restriction to I of the canonical map $\pi: T(\mathfrak{l}) \rightarrow U(\mathfrak{l})$. 
Let $\left\{x_{i}\right\}(i \in \Lambda)$ be a homogeneous basis of $\mathfrak{l}$, where $\Lambda$ is a totally order set. Let $p \in \mathbb{Z}_{+}$. By a $p$-tuple of $\Lambda$ we mean a sequence of $p$ elements of $\Lambda$. We write $\emptyset$ for the 0 -tuple. If $I$ is a $p$-tuple we set $y_{\mathbf{I}}=y_{\emptyset}=1$ in $U(\mathfrak{l})$, for $p=0$, and $y_{\mathbf{I}}=y_{i_{1}} \ldots y_{i p}$ in $U(\mathfrak{l})$ if $\mathbf{I}=\left(i_{1}, \ldots, i_{p}\right), i_{j} \in \Lambda, j=1, \ldots, p$, for $p \geqq 1$, where $y_{i}=\sigma\left(x_{i}\right)$ for all $i \in \Lambda$. A $p$-tuple of $\Lambda$ is said to be admissible if its elements are in nondecreasing order and if no index of an odd basis element appears more than once in the sequence.

Lemma 2.1 [1]. Let $\left\{x_{i}\right\}(i \in \Lambda)$ be an ordered basis of 1 . Then the monomials $y_{\mathbf{1}}$, for all the admissible $p$-tuples $\mathbf{I}$ of $\Lambda$, and all $p \geqq 0$, form a basis of $U(\mathfrak{l})$.

From Lemma 2.1 we immediately obtain the following:

Proposition 2.2. The canonical map $\sigma: \mathfrak{g} \rightarrow U(\mathfrak{g})$ is injective.

Proposition 2.2 allows us to identify $x_{i}$ and $y_{i}$. Now set $x_{\mathbf{I}}=x_{i_{1}} \ldots x_{i_{j}}$ for $I=\left(i_{1}, \ldots, i_{j}\right), j \geqq 1, x_{\emptyset}=1$. By Lemma 2.1 we have

Theorem 2.3 [1]. Let $\left\{x_{i}\right\}(i \in \Lambda)$ be an ordered basis of I. The monomials $x_{\mathbf{I}}$ for all admissible $p$-tuples $\mathbf{I}$ of $\Lambda$, and all $p \geqq 0$, form a basis of $U(\mathfrak{l})$.

Next, we will state the Poincaré-Birkhoff-Witt theorem for $\mathbb{Z}_{2}$-graded Lie algebras. First, we need a few more definitions.

An associative $\mathbb{Z}_{2}$-graded algebra $\mathfrak{a}$ is said to be commutative if $[\mathfrak{a}, \mathfrak{a}]=0$. The symmetric algebra of a $\mathbb{Z}_{2}$-graded algebra $\mathfrak{l}$ is a pair $(S(l), \varrho)$, where $S(\mathfrak{l})$ is a commutative associative $\mathbb{Z}_{2}$-graded algebra and $\varrho: \mathfrak{l} \rightarrow S(\mathfrak{l})$ is a linear map preserving the $\mathbb{Z}_{2}$-grading, with the following property: Given any pair $(\mathfrak{a}, \tau)$ where $\mathfrak{a}$ is a commutative associative $\mathbb{Z}_{2}$-graded algebra and $\tau: \mathfrak{l} \rightarrow \mathfrak{a}$ is a linear map preserving the $\mathbb{Z}_{2}$-grading, there is a unique homomorphism $\psi: S(\mathfrak{l}) \rightarrow \mathfrak{a}$ such that $\tau=\psi \circ \varrho$. Concretely, $S(\mathfrak{l})=T(\mathfrak{l}) / J$, where $J$ is the two-sided $\left(\mathbb{Z}_{2}\right.$-graded $)$ ideal in $T(\mathfrak{l})$ generated by the elements of the form $X \otimes Y-(-1)^{\alpha \beta} Y \otimes X, X \in \mathfrak{l}_{\alpha}, Y \in \mathfrak{l}_{\beta}$, $\alpha, \beta \in \mathbb{Z}_{2}$, and $\varrho$ is induced by the restriction to $\mathfrak{I}$ of the canonical map $\varepsilon: U(\mathfrak{l}) \rightarrow S(\mathfrak{l})$. It is clear that $\varepsilon$ maps I injectively. For this reason we will identify $\varepsilon(\mathfrak{l})$ with $\mathfrak{l}$.

We define a filtration of $T(\mathfrak{l})$ by $T_{p}(\mathfrak{l})=T^{0}(\mathfrak{l}) \oplus \ldots \oplus T^{p}(\mathfrak{l})$, and let $U_{p}(\mathfrak{l})=\pi\left(T_{p}(\mathfrak{l})\right)$ [respectively $S_{p}(\mathrm{l})=\varepsilon\left(T_{p}(\mathrm{l})\right)$ ] be the corresponding filtration of $U(\mathrm{l})$ [respectively of $S(\mathrm{l})]$. Consider the $\mathbb{Z}_{2}$-graded vector spaces $G^{p}(\mathrm{l})=U_{p}(\mathrm{l}) / U_{p-1}(\mathrm{l})$ and $G(\mathrm{l})$ $=\bigoplus_{i=0}^{\infty} G^{i}(\mathfrak{l})$, where we have set $U_{-1}(\mathfrak{l})=(0)$. Since $U_{p}(\mathfrak{l}) U_{q}(\mathfrak{l}) \subset U_{p+q}(\mathfrak{l})$, the multiplication in $U(\mathfrak{l})$ induces a multiplication in $G(\mathfrak{l})$. The resulting associative $\mathbb{Z}_{2}$-graded algebra is called the graded associative $\mathbb{Z}_{2}$-graded algebra associated to the filtered $\mathbb{Z}_{2}$-graded algebra $U(\mathrm{l})$.

Clearly, $G^{0}(\mathfrak{l})=U^{0}(\mathfrak{l})=\mathbb{C} \cdot 1$ and $\mathfrak{l}$ is canonically identified with $G^{1}(\mathfrak{l})$ thanks to Proposition 2.2. Since

$$
X Y=(-1)^{\alpha \beta} Y X+[X, Y]
$$

in $U_{2}(\mathfrak{l}), X \in \mathfrak{l}_{\alpha}, Y \in \mathfrak{l}_{\beta}, \alpha, \beta \in \mathbb{Z}_{2}$, we conclude that there is a unique homomorphism $\psi: S(\mathfrak{l}) \rightarrow G(\mathfrak{l})$ such that $\left.\psi\right|_{\mathbb{I}}=$ identity. [Here, we have identified $\mathbb{l}$ with its image in $S(\mathfrak{l})$.] $\psi$ is called the canonical homomorphism of $S(\mathfrak{l})$ in $G(\mathfrak{l})$. The next theorem is readily obtained from Theorem 2.3 . 
Theorem 2.4 (Poincaré-Birkhoff-Witt) [1]. The canonical homomorphism of $S(\mathfrak{l})$ into $G(\mathfrak{l})$ is an isomorphism.

Remark. The Poincaré-Birkhoff-Witt Theorem for $\mathbb{Z}$-graded Lie algebras was first proved in [18].

Corollary 2.5. Let $\mathfrak{H} \subset \mathfrak{l}$ be a subalgebra of $\mathfrak{l}$ with an ordered basis $\left\{x_{i}\right\}_{i \in M}$. Let $\left\{x_{i}\right\}_{i \in M \cup N}$ be an ordered basis of $\mathfrak{l}$ extending $\left\{x_{i}\right\}_{i \in M}$, and such that $i<j$ for $i \in M$, $j \in N$. Then the inclusion $\mathfrak{f} \subset \mathbb{I}$ induces an injective homomorphism $U(\mathfrak{l}) \rightarrow U(\mathfrak{l})$, and $U(\mathfrak{l})$ is a free $U(\mathfrak{l})$-module with basis $\left\{x_{I}\right\}$ for all admissible $p$-tuples $\mathbf{I}$ of $N$, all $p \geqq 0$.

For a $\mathbb{Z}_{2}$-graded vector space $V=V_{\overline{0}} \oplus V_{\overline{1}}$ we let $\operatorname{End}(V)$ be the associative $\mathbb{Z}_{2}$-graded algebra of (graded) endomorphisms, $\operatorname{End}(V)=(\operatorname{End}(V))_{\overline{0}} \oplus(\operatorname{End}(V))_{\overline{1}}$, where

$$
(\operatorname{End}(V))_{\alpha}=\left\{T \in \operatorname{End}(V) \mid T\left(V_{\gamma}\right) \subset V_{\alpha+\beta} \text { for all } \gamma \in \mathbb{Z}_{2}\right\},
$$

$\alpha \in \mathbb{Z}_{2}$. We set $\mathfrak{I}(V)=(\operatorname{End}(V))_{L}$. A representation $\pi$ of a $\mathbb{Z}_{2}$-graded Lie algebra $\mathrm{I}$ is a homomorphism $\pi: \mathfrak{l} \rightarrow \mathfrak{l}(V)$. We set $X v=\pi(X) v$, for $X \in \mathfrak{l}, v \in V$. It is clear from the definition that

$$
X\left(V_{\beta}\right) \subset V_{\alpha+\beta} \text { for all } X \in \mathfrak{l}_{\alpha}, \alpha \in \mathbb{Z}_{2},
$$

and that

$$
[X, Y] v=X Y v-(-1)^{\alpha \beta} Y X v, \text { for all } X \in \mathfrak{l}_{\alpha}, Y \in \mathfrak{I}_{\beta}, \alpha, \beta \in \mathbb{Z}_{2} .
$$

We will also use the terminologies of " $U(\mathfrak{l})$-module" and "I-module" in place of "representation of I." A submodule of an l-module is always meant to be $\mathbb{Z}_{2}$-graded, and a homomorphism $\Phi: M \rightarrow M^{\prime}$ of l-modules, means one such that $\Phi\left(M_{\alpha}\right) \subset M_{\psi(\alpha)}^{\prime}$, where $\psi: \mathbb{Z}_{2} \rightarrow \mathbb{Z}_{2}$ is a bijection.

Let $\mathfrak{l} C \mathfrak{l}$ be a subalgebra of $\mathfrak{l}, W$ a $\mathfrak{l}$-module. Thanks to Corollary 2.5 we can view $U(\mathfrak{l})$ as a subalgebra of $U(\mathfrak{l})$. One can consider $U(\mathfrak{l})$ as a right $U(\mathfrak{l})$-module and therefore may form the left $U(\mathfrak{l})$-module $V=U(\mathfrak{l}) \otimes_{U(\mathfrak{l})} W$. I.e., let $M$ be the $\mathbb{Z}_{2}$-graded subspace of $U(\mathfrak{l}) \otimes W$ generated by the elements of form $X Y \otimes v$ $-X \otimes Y v$ for all $X \in U(\mathfrak{l}), Y \in U(\mathfrak{f})$ and $v \in W$. Then $V$ is the $\mathbb{Z}_{2}$-graded space $(U(\mathfrak{l}) \otimes W) / M . V$ is made into a $U(\mathfrak{l})$-module by setting

$$
X(Y \otimes v)=X Y \otimes v, X, Y \in U(\mathrm{l}), v \in W .
$$

$V$ is called the $U(\mathfrak{l})$-module induced from the $U(\mathfrak{f})$-module $W$ and denoted $\operatorname{Ind}_{\mathfrak{f}}(W)$. By Corollary 2.5 the homomorphism $w \mapsto 1 \otimes w$ from $W$ to $V$ is injective. We identify $W$ with $1 \otimes W$ by this map.

Proposition 2.6. Let $\mathfrak{f} \subset \mathfrak{l}$ be a subalgebra of $\mathfrak{l}, W$ a $\mathfrak{\mathfrak { l }}$-module, $V=\operatorname{Ind}_{\mathfrak{f}}^{\mathrm{I}}(W)$ and $V^{\prime}$ and l-module. Any $\mathfrak{l}$-homomorphism $\psi: W \rightarrow V^{\prime}$ can be uniquely extended to an I-homomorphism $\phi: V \rightarrow V^{\prime}$. Furthermore, $\psi \mapsto \phi$ is a bijection from $\operatorname{Hom}_{\mathfrak{f}}\left(W, V^{\prime}\right)$ to $\operatorname{Hom}_{\mathfrak{l}}\left(V, V^{\prime}\right)$.

Proof. Let $\phi(X \otimes w)=X \psi(w)$. If $X \in U(\mathfrak{l}), Y \in U(\mathfrak{f})$, then $\phi(X Y \otimes w-X \otimes Y w)$ $=X Y \psi(w)-X \psi(Y w)=0$, so $\phi$ is a homomorphism from $V$ to $V^{\prime}$. The restriction of $\phi$ to $1 \otimes W$ gives a map from $\operatorname{Hom}_{\mathfrak{l}}\left(V, V^{\prime}\right)$ to $\operatorname{Hom}_{\mathfrak{f}}\left(W, V^{\prime}\right)$ inverse to $\psi \mapsto \phi$. Q.E.D. 


\section{Highest Weight Representations}

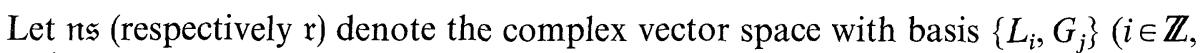
$\left.j \in \frac{1}{2}+\mathbb{Z}\right)\left(\right.$ respectively $\left\{L_{i}, G_{j}\right\}(i, j \in \mathbb{Z})$ ), and set

$$
\begin{aligned}
& {\left[L_{i}, L_{j}\right]=(j-i) L_{i+j},} \\
& {\left[G_{i}, G_{j}\right]=-2 L_{i+j},} \\
& {\left[L_{i}, G_{j}\right]=\left(j-\frac{1}{2} i\right) G_{i+j} .}
\end{aligned}
$$

Let $\mathfrak{v}=\bigoplus_{i \in \mathbb{Z}} \mathbb{C} L_{i}$, and set $\widehat{\mathfrak{r}}_{\overline{0}}=r_{\overline{0}}=\mathfrak{v}, \widehat{\mathfrak{r}}_{\overline{1}}=\bigoplus_{i \in \frac{1}{2}+\mathbb{Z}} \mathbb{C} G_{i} \cdot \mathfrak{r}_{\overline{1}}=\bigoplus_{i \in \mathbb{Z}} \mathbb{C} G_{i}$. It is easy to see that $\mathfrak{n s}$ and $\mathfrak{r}$ are $\mathbb{Z}_{2}$-graded Lie algebras and that $\mathfrak{v}$ is a subalgebra of $\mathfrak{n s}$ and $\mathfrak{r} . \mathfrak{v}$ is a Lie algebra in the usual sense. Now let

$$
\begin{aligned}
& C\left(L_{i}, L_{j}\right)=\frac{1}{8} \delta_{i,-j}\left(i^{3}-i\right), \\
& C\left(G_{i}, G_{j}\right)=\frac{1}{2} \delta_{i,-j}\left(i^{2}-\frac{1}{4}\right), \\
& C\left(L_{i}, G_{j}\right)=0 .
\end{aligned}
$$

It is also easy to see that $C$ satisfies the cocycle condition for $\mathbb{Z}_{2}$-graded Lie algebras. The central extension ${ }^{3}$ of $n \mathfrak{s}$ (respectively $\mathfrak{r}$ ) corresponding to the cocycle $C$ is called the Neveu-Schwarz (respectively Ramond) algebra and is denoted by $\hat{\imath}$ (respectively $\hat{\mathfrak{r}}$ ) (See Appendix for the definitions of cocycle condition and central extension corresponding to a cocycle, for $\mathbb{Z}_{2}$-graded Lie algebras.) The central extension of $\mathfrak{v}$ corresponding to $\left.C\right|_{\mathfrak{v} \times \mathfrak{v}}$ is called the Virasoro algebra and is denoted by $\hat{\mathfrak{v}}$.

From now on, in this section, we let $\mathfrak{g}$ denote either one of the $\mathbb{Z}_{2}$-graded Lie algebras $\hat{\boldsymbol{r}}$ and $\hat{\mathfrak{x}}$. The $\mathbb{Z}_{2}$-grading is given by

$$
g_{\overline{0}}=\left(\bigoplus_{i \in \mathbb{Z}} \mathbb{C} e_{i}^{+}\right) \oplus \mathbb{C} e_{0}^{\prime}, \mathfrak{n s}_{\overline{1}}=\bigoplus_{i \in \frac{1}{2}+\mathbb{Z}} \mathbb{C} e_{i}^{-}, \mathfrak{r}_{\overline{1}}=\bigoplus_{i \in \mathbb{Z}} \mathbb{C} e_{i}^{-},
$$

where $e_{i}^{+}=\left(L_{i}, 0\right), e_{i}^{-}=\left(G_{i}, 0\right)$, and $e_{0}^{\prime}=(0,1)$ in the notation of the Appendix. (We sometimes drop the superscripts of $e_{i}^{+}, e_{i}^{-}$if $\mathfrak{g}=\widehat{n} \hat{s}$.)

If $I$ is a Lie algebra in the usual sense, then $U(l)$ is isomorphic to a polynomial ring. Therefore, by the P-B-W theorem, $U(\mathfrak{l})$ is an integral domain. If $\mathfrak{l}$ is a $\mathbb{Z}_{2}$-graded Lie algebra, however, $S(l)$ is not in general isomorphic with a polynomial ring. Nevertheless one has the following

Proposition 3.1. $U(\mathfrak{g})$ is an integral domain.

Proof. We let $B=\left\{e_{n} \mid n \in \mathbb{Z}\right\} \cup\left\{e_{m} \mid m \in \mathbb{Z}+\frac{1}{2}\right\} \cup\left\{e_{0}^{\prime}\right\}$ if $\mathfrak{g}=\hat{\mathfrak{r}}$, and $B=\left\{e_{n}^{+} \mid n \in \mathbb{Z}\right\}$ $\cup\left\{e_{m}^{-} \mid m \in \mathbb{Z}\right\} \cup\left\{e_{0}^{\prime}\right\}$ if $\mathfrak{g}=\hat{\mathfrak{r}}$. We enumerate the elements of $B$ by $X: \mathbb{Z} \rightarrow B$, where $X(0)=e_{0}^{\prime}, \quad X(2 n-1)=e_{(2 n-1) / 2}, \quad X(2 n)=e_{n}, \quad X(-2 n+1)=e_{1-n}, \quad X(-2 n)$ $=e_{-(2 n-1) / 2}, \quad n \in \mathbb{N}$, if $\mathfrak{g}=\hat{\mathfrak{H} s}$, and $X(0)=e_{0}^{\prime} \quad X(2 n-1)=e_{n}^{-}, \quad X(2 n)=e_{n-1}^{+}$, $X(-2 n+1)=e_{-n}^{+}, \quad X_{(-2 n)}=e_{1-n}^{-}, \quad n \in \mathbb{N}$, if $\mathfrak{g}=\hat{\mathfrak{r}}$. Let $x_{m}=X(m), \quad m \in \mathbb{Z}$. By Theorem 2.3, a basis of $U(\mathfrak{g})$ consists of the monomials $x_{\mathbf{I}}=x_{i_{1}} \ldots x_{i_{m}}$, where $\mathbf{I}=\left(i_{1}, \ldots, i_{m}\right)$ is an admissible $m$-tuple of $\mathbb{Z}$. If $x_{j} \in \mathfrak{g}_{\overline{0}}$ and $\mathbf{I}=\left(i_{1}, \ldots, i_{m}\right)$ is an

\footnotetext{
${ }^{3}$ By Proposition A.2 and the results of [21] one sees that these are universal central extensions
} 
admissible $m$-tuple of $\mathbb{Z}$, let $Z(j, \mathbf{I})$ denote the set of all $i_{k}, k=1, \ldots, m$, such that either $i_{k}=j$ or $x_{i k} \in \mathfrak{g}_{\overline{1}}$ and $\left[x_{i_{k}}, x_{i_{k}}\right] \in-2 x_{j}+\mathbb{C} x_{0}$. For $i \in \mathbb{Z}$ set $a_{i}=2$ if $x_{i} \in \mathfrak{g}_{\overline{0}}$ and $a_{i}=1$ if $x_{i} \in \mathfrak{g}_{\overline{1}}$. We define the $j$-degree of $x_{\mathbf{I}}$ as $j-\operatorname{deg}\left(x_{\mathbf{I}}\right)=0$ if $Z(j, \mathbf{I})=\emptyset$ and $j-\underset{m}{\operatorname{deg}}\left(x_{\mathbf{I}}\right)=\sum_{i_{k} \in \mathbb{Z}(j, \mathbf{I})} a_{i_{k}}$, otherwise. We define the total degree of $x_{\mathbf{I}}$ as $t-\operatorname{deg}\left(x_{\mathbf{I}}\right)$ $=\sum_{k=1}^{m} a_{i_{k}}$. Note that $t-\operatorname{deg}\left(x_{\mathbf{I}}\right)=\sum_{j \mid x_{j} \in \mathfrak{g} 0} j-\operatorname{deg}\left(x_{\mathbf{I}}\right)$. Given $\mathbf{I}=\left(i_{1}, \ldots, i_{m}\right)$ and $\mathbf{J}=\left(j_{1}, \ldots, j_{n}\right), \mathbf{I} \neq \mathbf{J}$ such that $t-\operatorname{deg}\left(x_{\mathbf{I}}\right)=t-\operatorname{deg}\left(x_{\mathbf{J}}\right)$, we choose the leading monomial between $x_{\mathbf{I}}$ and $x_{\mathbf{J}}$ as follows: Let $j_{0}$ be the largest even index (i.e. $x_{j_{0}} \in \mathfrak{g}_{\overline{0}}$ ) such that $j_{0}-\operatorname{deg}\left(x_{\mathbf{I}}\right) \neq j_{0}-\operatorname{deg}\left(x_{\mathbf{J}}\right)$. The leading monomial is the one with greatest $j_{0}$-degree. If $x=\sum_{\mathbf{I}} a_{\mathbf{I}} x_{\mathbf{I}}$ we set $t-\operatorname{deg}(x)=\max _{\mathbf{I}}\left\{t-\operatorname{deg}\left(x_{\mathbf{I}}\right)\right\}$. If $t-\operatorname{deg}(x)=p$, the leading monomial of $x$ is, by definition, the leading monomial of total degree $p$. We note: 1$) t-\operatorname{deg}\left(\left[x_{i}, x_{j}\right]\right)<t-\operatorname{deg}\left(x_{i} x_{j}\right)$ if $x_{i}$ or $x_{j} \in \mathfrak{g}_{\overline{0}}$. 2) Let $x_{i}, x_{j} \in \mathfrak{g}_{\overline{1}}, i \neq j$, and say $i<j$. There are $k, k^{\prime} \in \mathbb{Z}$ such that $\left[x_{j}, x_{j}\right]=-2 x_{k}+c_{j} x_{0}$ and $\left[x_{i}, x_{j}\right]=-2 x_{k^{\prime}}$ $+c_{i j} x_{0}$. Clearly $k>k^{\prime}$. If $c_{j} \neq 0$, then $c_{i j}=0$, and if $c_{i j} \neq 0$, then $c_{j}=0$ and $k>0$. Now, $k-\operatorname{deg}\left(x_{i} x_{j}\right)=1$. This implies that $x_{i} x_{j}$ leads the terms of $\left[x_{i}, x_{j}\right]$ if $i \neq j$. 3) $x_{i}^{2}$ $=\frac{1}{2}\left[x_{i}, x_{i}\right]=-x_{j}+c_{i} x_{0}$, where $x_{j} \in \mathfrak{g}_{\overline{0}}$, if $x_{i} \in \mathfrak{g}_{\overline{1}}$. Let $a_{\mathbf{K}} x_{\mathbf{K}}$ be the leading monomial among the monomials of $x_{\mathbf{I}} x_{\mathbf{J}}$ obtained by bracketing only an odd element with itself. By 1)-3) $t-\operatorname{deg}\left(x_{\mathbf{I}} x_{\mathbf{J}}\right)=t-\operatorname{deg}\left(a_{\mathbf{K}} x_{\mathbf{K}}\right)=t-\operatorname{deg}\left(x_{\mathbf{I}}\right)+t-\operatorname{deg}\left(x_{\mathbf{J}}\right)$, $j-\operatorname{deg}\left(a_{\mathbf{K}} x_{\mathbf{K}}\right)=j-\operatorname{deg}\left(x_{\mathbf{I}}\right)+j-\operatorname{deg}\left(x_{\mathbf{J}}\right)$ for all $j$ such that $x_{j} \in \mathfrak{g}_{\overline{0}}$, so that $a_{\mathbf{K}} x_{\mathbf{K}}$ is the leading term of $x_{\mathbf{I}} x_{\mathbf{J}}$. Now, if $x_{\mathbf{I}}$ is the leading monomial of $x$ and $y_{\mathbf{J}}$ is the leading monomial of $y$, then the leading monomial of $x_{\mathbf{I}} x_{\mathbf{J}}$ constructed above is the leading term of $x y$. Hence $x y \neq 0$ if $x \neq 0, y \neq 0$. Q.E.D.

Let $\mathfrak{h}=\mathbb{C} e_{0}^{+} \oplus \mathbb{C} e_{0}^{\prime} \subset \mathfrak{g}$. We define $\delta \in \mathfrak{h} *$ by requiring that $\delta\left(e_{0}\right)=1$ and $\delta\left(e_{0}^{\prime}\right)=0$, if $\mathfrak{g}=\hat{\mathfrak{r}} \mathfrak{s}$, and that $\delta\left(e_{0}^{+}\right)=1$ and $\delta\left(e_{0}^{\prime}\right)=0$, if $\mathfrak{g}=\hat{\mathfrak{r}}$. Let $Q=\frac{1}{2} \mathbb{Z} \delta$, if $\mathfrak{g}=\hat{\mathfrak{r}} \hat{\mathfrak{s}}$, and $Q=\mathbb{Z} \delta$, if $\mathrm{g}=\mathrm{r}$. For $\eta \in Q$, let

$$
\mathfrak{g}^{\eta}=\{X \in \mathfrak{g} \mid[H, X]=\eta(H) X \text { for all } H \in \mathfrak{h}\} .
$$

We then have $\widehat{n \mathfrak{s}}^{0}=\mathbb{C} e_{0} \oplus \mathbb{C} e_{0}^{\prime}$ and $\mathfrak{n s}^{i \delta}=\mathbb{C} e_{i}$ for all $i \in \mathbb{Z} \backslash\{0\}$, $\hat{\mathfrak{r}}^{0}=\mathbb{C} e_{0}^{+} \oplus \mathbb{C} e_{0}^{-} \oplus \mathbb{C} e_{0}^{\prime}$ and $\hat{\mathfrak{r}}^{i \delta}=\mathbb{C} e_{i}^{+} \oplus \mathbb{C} e_{i}^{-}$, for all $i \in \mathbb{Z} \backslash\{0\}$. Hence $\mathfrak{g}=\bigoplus_{\eta \in Q} \mathfrak{g}^{\eta}$ and $\left[\mathfrak{g}^{\eta_{1}}, \mathfrak{g}^{\eta_{2}}\right] \subset \mathfrak{g}^{\eta_{1}+\eta_{2}}$ for all $\eta_{1}, \eta_{2} \in Q$. We write $i \delta>0$ (respectively $i \delta<0$ ) if $i>0$ (respectively $i<0$ ), where $i \in \frac{1}{2} \mathbb{Z}$. Let $Q^{+}$denote the set of all $\eta \in Q$ such that $\eta>0$. We define the subalgebras $n$ and $n^{-}$of $\mathfrak{g}$ as $n=\bigoplus_{\eta \in Q^{+}} \mathfrak{g}^{\eta}$ and $\boldsymbol{n}^{-}=\bigoplus_{-\eta \in Q^{+}} \mathfrak{g}^{\eta}$. Then $\mathfrak{g}=\mathfrak{n}^{-} \oplus \mathfrak{g}^{0} \oplus \mathfrak{n}$. Let $\mathfrak{b}=\mathfrak{g}^{0} \oplus \mathfrak{n}$. If $M$ is an $\mathfrak{h}$-module, and $\mu \in \mathfrak{h}^{*}$, let

$$
M^{\mu}=\{v \in M \mid H v=\mu(H) v \text { for all } H \in \mathfrak{h}\} .
$$
Definition 3.2. A $\mathfrak{g}$-module $M$ is said to be a weight module if $M=\bigoplus_{\mu \in \mathfrak{h}^{*}} M^{\mu}$, with
$\operatorname{dim} M^{\mu}<\infty$ for all $\lambda \in \mathfrak{h}^{*} . M^{\mu}$ is called the $\mu$-weightspace of $M$.

Definition 3.3. Let $M$ be a $\mathfrak{g}$-module, $\mu \in \mathfrak{h}^{*}, v \in M_{\overline{0}} \backslash\{0\}$ such that $H v=\lambda(H) v$ for all $H \in \mathfrak{h}, \mathfrak{n} v=0$ and $M=U(\mathfrak{g}) v$. Then $M$ is said to be a highest weight module with highest weight $\lambda$.

Remark. Any highest weight module is a weight module with weightspace

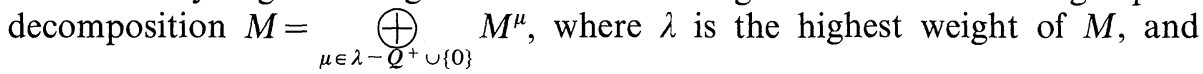


$M^{\lambda}=\mathbb{C} v, \quad M^{\lambda-\eta}=U\left(\mathfrak{n}^{-}\right)^{-\eta} v, \quad$ if $\quad \mathfrak{g}=\hat{\mathfrak{r s}}, \quad$ and $\quad M^{\lambda}=\mathbb{C} v \oplus \mathbb{C} e_{0}^{-} v, \quad M^{\lambda-\eta}$ $=\left(U\left(\mathrm{n}^{-}\right) \oplus U\left(\mathrm{n}^{-}\right) e_{0}^{-}\right)^{-\eta} v$ if $\mathfrak{g}=\hat{\mathfrak{x}}$. Here $U\left(\mathrm{n}^{-}\right)^{-\eta}$ and $\left(U\left(\mathrm{n}^{-}\right) \oplus U\left(\mathrm{n}^{-}\right) e_{0}^{-}\right)^{-\eta}$ are the weightspaces of $U\left(\mathrm{n}^{-}\right)$and $U\left(\mathrm{n}^{-}\right) \oplus U\left(\mathrm{n}^{-}\right) e_{0}^{-}$, respectively, viewed as $\mathfrak{h}$-modules, and $M=U(\mathfrak{g}) v$. Any nonzero homogeneous element of $M^{\lambda}$ is called a highest weight vector.

Let $\lambda \in \mathfrak{h}^{*}$ and let $\lambda\left(e_{0}^{+}\right)=h, \lambda\left(e_{0}^{\prime}\right)=c$. If $\mathfrak{g}=\hat{\mathfrak{r s}}$, let $\mathbb{C}(\lambda)$ denote the onedimensional even $\mathfrak{b}$-module $\mathbb{C}$ defined by $e_{0} 1=h 1, e_{0}^{\prime} 1=c 1, \boldsymbol{n} 1=0, \operatorname{deg} 1=\overline{0}$. If $\mathfrak{g}=\hat{\mathfrak{r}}$ we denote by $R(\lambda)$ the two-dimensional $\mathbb{Z}_{2}$-graded $\mathfrak{b}$-module generated by $v_{\lambda}$, where $\mathfrak{n} \cdot v_{\lambda}=0, e_{0}^{+} v_{\lambda}=h v_{\lambda}, e_{0}^{\prime} v_{\lambda}=c v_{\lambda}, \operatorname{deg}\left(v_{\lambda}\right)=\overline{0}$. If $\mathfrak{g}=\hat{\mathfrak{x}}$, and $\lambda$ is such that $h=-\frac{c}{16}$, we denote by $S(\lambda)$ the one-dimensional even $\mathfrak{b}$-module $\mathbb{C}$ defined by $\mathrm{n} 1=0, e_{0}^{+} 1=h 1, e_{0}^{\prime} 1=c 1, e_{0}^{-} 1=0, \operatorname{deg} 1=\overline{0}$. If $h \neq-\frac{c}{16}$ thus $R(\lambda)$ is irreducible (as a $\mathbb{Z}_{2}$-graded $\mathfrak{b}$-module). If $h=-\frac{c}{16}$, then $e_{0}^{-} v_{\lambda}$ generates a proper $U(\mathfrak{b})$-submodule of $R(\lambda)$ isomorphic to $S(\lambda)$

Definition 3.4. Let $\lambda \in \mathfrak{h}^{*}$, and let $V(\lambda)$ be one of the $\mathfrak{b}$-modules $\mathbb{C}(\lambda), R(\lambda)$, or $S(\lambda)$ if $\lambda\left(e_{0}^{+}\right)=-\lambda\left(e_{0}^{\prime}\right) / 16$. Set $M(V(\lambda))=\operatorname{Ind}_{b}^{\mathfrak{g}}(V(\lambda))$. If $V(\lambda)=\mathbb{C}(\lambda)$ or $R(\lambda), M(V(\lambda))$ is called the Verma module associated with $\mathfrak{g}, \mathfrak{h}$, and $\lambda$. If $\lambda\left(e_{0}^{+}\right)=-\lambda\left(e_{0}^{\prime}\right) / 16, M(S(\lambda))$ is called the half Verma module associated with $\mathfrak{g}, \mathfrak{h}$, and $\lambda$. (This terminology will be justified by Proposition 3.12.)

Remark. $M(V(\lambda))=U\left(\mathrm{n}^{-}\right) \otimes_{\mathbb{C}} V(\lambda)$ by Corollary 2.5. We have $M(V(\lambda))^{\lambda-\eta}$

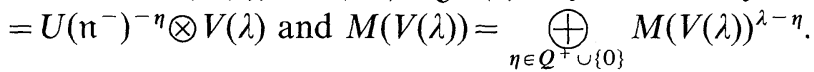

The following is an immediate consequence of the universal property of the tensor product.

Proposition 3.5. If $\lambda\left(e_{0}^{+}\right)=-\lambda\left(e_{0}^{\prime}\right) / 16$, then $M(S(\lambda))$ is a submodule of $M(R(\hat{\lambda}))$.

We denote by $p(n)$ the number of partitions of $n$ and by $p(S, n)$ the number of partitions of $n$ that belong to the subset $S$ of the set of all partitions. For a subset $H$ of the set of positive integers, we denote by " $H$ " the set of all partitions with parts in $H$, and by " $H$ " $(\leqq d)$ the set of all partitions with parts in $H$, in which no part appears more than $d$ times. Let $H_{0}$ (respectively $H_{1}$ ) denote the even (respectively odd) positive integers. Finally, let $p_{e}(\mathscr{D}, n)\left[\right.$ respectively $\left.p_{o}(\mathscr{D}, n)\right)$ be the number of partitions of $n$ into an even (respectively odd) number of distinct parts, and let $p(\mathscr{D}, n)$ be the number of partitions of $n$ into distinct parts.

If $M$ is a weight module we set $M_{\gamma}^{\eta}=M^{\mu} \cap M_{\gamma}, \mu \in \mathfrak{h} *, \gamma \in \mathbb{Z}_{2}$.

Proposition 3.6. Let $\lambda \in \mathfrak{h}^{*}$,

$$
\operatorname{dim} M(\mathbb{C}(\lambda))^{\lambda-n \delta}=\hat{p}(n)=\tilde{p}(2 n),
$$

where

$$
\begin{aligned}
\tilde{p}(0)=1, \tilde{p}(s)= & \sum_{i=0}^{s} p\left(“ H_{0} ", i\right) p\left(“ H_{1} "(\leqq 1), s-i\right), s>0 \\
& \operatorname{dim} M(R(\lambda))_{\gamma}^{\lambda-n \delta}=\hat{p}(n),
\end{aligned}
$$

where

$$
\hat{p}(0)=1, \hat{p}(n)=\sum_{i=0}^{n} p(i) p(\mathscr{D}, n-i), n>0, \gamma \in \mathbb{Z}_{2}
$$




$$
\text { If } \begin{aligned}
\lambda\left(e_{0}\right)= & -\lambda\left(e_{0}^{+}\right) / 16, \text { then } \\
& \left.\operatorname{dim} M(S(\lambda))_{\gamma}^{\lambda-n \delta}=\frac{1}{2} \hat{p}(n)\right) \quad \text { for } n>0, \hat{p}(n) \text { as in }(2) .
\end{aligned}
$$

Proof. By the remark that follows Definition 3.4, $M(R(\lambda))^{\lambda-n \delta}$ is a linear combination of elements

$$
e_{-i_{1}}^{+} \ldots e_{-i_{r}}^{+} e_{-j_{1}}^{-} \ldots e_{-j_{s}}^{-} \otimes v_{\lambda}, e_{-k_{1}}^{+} \ldots e_{-k_{t}}^{+} e_{-l_{1}}^{-} \ldots e_{-l_{u}}^{-} \otimes e_{0}^{-} v_{\lambda},
$$

where

$$
\begin{gathered}
i_{1}+\ldots+i_{r}+j_{1}+\ldots+j_{s}=k_{1}+\ldots+k_{t}+l_{1}+\ldots+l_{u}=n, \\
i_{1} \geqq \ldots \geqq i_{r}, j_{1}>\ldots>j_{s}, k_{1} \geqq \ldots \geqq k_{t}, l_{1}>\ldots>l_{u},
\end{gathered}
$$

$s$ even, $u$ odd. Hence,

$$
\begin{aligned}
\operatorname{dim} M(R(\lambda))_{0}^{\frac{\lambda}{0}-n \delta} & =\sum_{i=0}^{n} p(i) p_{e}(\mathscr{D}, n-i)+\sum_{i=0}^{n} p(i) p_{o}(\mathscr{D}, n-i) \\
& =\sum_{i=0}^{n} p(i) p(\mathscr{D}, n-i) .
\end{aligned}
$$

Similarly, for $M(R(\lambda)) \frac{\lambda-n \delta}{1}$. This proves (2).

To prove (3) we use a similar argument and note that

$$
\sum_{i=0}^{n} p(i) p_{e}(\mathscr{D}, n-i)=\sum_{i=0}^{n} p(i) p_{o}(\mathscr{D}, n-i), \text { for all } n>0,
$$

since

$$
\left(\sum_{n=0}^{\infty} p(n) q^{n}\right)\left(\sum_{n=0}^{\infty}\left(p_{e}(\mathscr{D}, n)-p_{o}(\mathscr{D}, n)\right) q^{n}\right)=1
$$

(see, e.g. [10]). (1) is even easier. Q.E.D.

Let $v_{\lambda}=1 \otimes v_{\lambda}$ in $M(R(\lambda))$ and $v_{\lambda}=1 \otimes 1$ in $M(\mathbb{C}(\lambda))$ or in $M(S(\lambda))$.

Proposition 3.7. Let $M$ be a highest weight module with highest weight $\lambda$ and let $v$ be a generating highest weight vector of $M$. If $\mathfrak{g}=\hat{\mathfrak{r} s}$, we let $V(\lambda)=\mathbb{C}(\lambda)$; if $\mathfrak{g}=\hat{\mathfrak{r}}$ we let $V(\lambda)=R(\lambda)$ if $e_{0}^{-} v \neq 0$, and $V(\lambda)=S(\lambda)$ if $e_{0}^{-} v=0$ (in which case $\lambda\left(e_{0}^{+}\right)$ $\left.=-\lambda\left(e_{0}^{\prime}\right) / 16\right)$. Then there is a unique surjective homomorphism $\phi: M(V(\lambda)) \rightarrow M$ such that $\phi\left(v_{\lambda}\right)=v$.

Proof. This is immediate from Proposition 2.6. Q.E.D.

Proposition 3.8. Let $M$ be a highest weight module. Then $M$ has a unique maximal proper submodule.

Proof. Let $\lambda$ be the highest weight of $M$. If $g=\widehat{\pi} \mathfrak{s}$, or if $\mathfrak{g}=\hat{\mathfrak{x}}$ and $\lambda\left(e_{0}^{+}\right) \neq \lambda\left(e_{0}^{\prime}\right) / 16$, then every proper submodule of $M$ is contained in $\bigoplus_{n>0} M^{\lambda-n \delta}$. If $\mathfrak{g}=\hat{\mathfrak{r}}$ and $\lambda\left(e_{0}^{+}\right)$ $=-\lambda\left(e_{0}^{\prime}\right) / 16$, then every proper submodule of $M$ is contained in $\bigoplus_{n>0} M^{\lambda-n \delta} \oplus M_{1}^{\lambda}$. In either case, the sum of all proper submodules is itself a proper submodule. Q.E.D. 
Definition 3.9. $L(V(\lambda))=$ unique irreducible quotient of $M(V(\lambda)), V(\lambda)=\mathbb{C}(\lambda)$, $R(\lambda)$ or $S(\lambda)$.

Proposition 3.10. Let $\lambda\left(e_{0}^{+}\right)=-\lambda\left(e_{0}^{\prime}\right) / 16$. Then $L(R(\lambda)) \simeq L(S(\lambda))$.

Proof. Let $M(R(\lambda))_{(1)}$ [respectively $\left.M(S(\lambda))_{(1)}\right]$ denote the unique maximal proper submodule of $M(R(\lambda))$ [respectively $M(S(\lambda))$ ]. By Proposition 3.5, $e_{0}^{-}(1 \otimes$ $\left.v_{\lambda}\right) \in M(R(\lambda))_{(1)}$. Therefore the image $\overline{1 \otimes v_{\lambda}}$ of $1 \otimes v_{\lambda}$ by the quotient map is annihilated by $e_{0}^{-}$. By Proposition 3.7, there is a unique surjective homomorphism $\phi: M(S(\lambda)) \rightarrow L(R(\lambda))$ such that $\phi(1 \otimes 1)=\overline{1 \otimes v_{\lambda}}$. This implies that

$$
L(R(\lambda)) \simeq M(S(\lambda)) / \operatorname{ker} \phi=L(S(\lambda)) . \quad \text { Q.E.D. }
$$

Definition 3.11. If $M$ is a weight module, we define the character of $M$ as

$$
\operatorname{ch} M=\sum_{\mu \in \mathfrak{h}^{*}}\left(\operatorname{dim} M^{\mu}\right) q^{\mu} .
$$

From now on we abbreviate $q^{n \delta}$ by $q^{n}, n \delta \in Q$.

Proposition 3.12. (1) $\operatorname{ch} M(\mathbb{C}(\lambda))=q^{\lambda} \frac{\prod_{n \in \mathbb{N}-1 / 2}\left(1+q^{-n}\right)}{\prod_{n \in \mathbb{N}}\left(1-q^{-n}\right)}$;

(2) $\operatorname{ch} M(R(\lambda))=2 q^{\lambda} \prod_{n \in \mathbb{N}} \frac{\left(1+q^{-n}\right)}{\left(1-q^{-n}\right)}$;

(3) $\operatorname{ch} M(S(\lambda))=q^{\lambda} \prod_{n \in \mathbb{N}} \frac{\left(1+q^{-n}\right)}{\left(1-q^{-n}\right)}$, if $\lambda\left(e_{0}^{+}\right)=-\lambda\left(e_{0}^{\prime}\right) / 16$.

Proof.

$$
\sum_{n \in \mathbb{Z}_{+}} \tilde{p}(n) q^{-n}=\prod_{n \text { even }}\left(1+q^{-n}+q^{-2 n}+\ldots\right) \prod_{n \text { odd }}\left(1+q^{-n}\right)=\frac{\prod_{n \text { odd }}\left(1+q^{-n}\right)}{\prod_{n \text { even }}\left(1-q^{-n}\right)},
$$

$\tilde{p}(n)$ as in Proposition 3.6. By Proposition 3.6,

$$
\operatorname{ch} M(\mathbb{C}(\lambda))=\sum_{n \in \frac{1}{2} \mathbb{Z}_{+}} \tilde{p}(2 n) q^{\lambda-n \delta}=q^{\lambda} \sum_{n \in \mathbb{Z}} \tilde{p}(n) q^{-n / 2}=q^{\lambda} \frac{\prod_{n \in \mathbb{N}-1 / 2}\left(1+q^{-n}\right)}{\prod_{n \in \mathbb{N}}\left(1-q^{-n}\right)}
$$

By Proposition 3.6,

$$
\begin{aligned}
\operatorname{ch} M(R(\lambda)) & =\sum_{n \in \mathbb{Z}_{+}} \operatorname{dim}\left(M(R(\lambda))^{\lambda-n \delta}\right) q^{\lambda-n \delta}=2 q^{\lambda} \sum_{n \in \mathbb{Z}_{+}} \hat{p}(n) q^{-n} \\
& =2 q^{\lambda} \prod_{n \in \mathbb{N}}\left(1+q^{-n}+q^{-2 n}+\ldots\right) \prod_{n \in \mathbb{N}}\left(1+q^{-n}\right)=2 q^{\lambda} \prod_{n \in \mathbb{N}} \frac{\left(1+q^{-n}\right)}{\left(1-q^{-n}\right)} .
\end{aligned}
$$

This proves (1) and (2). (3) follows from (2) and Proposition 3.6. Q.E.D.

Proposition 3.13. Let $V(\lambda)=\mathbb{C}(\lambda), V(\mu)=\mathbb{C}(\mu)$, or $V(\lambda)=R(\lambda), V(\mu)=R(\mu)$, or $V(\lambda)=S(\lambda), \quad V(\mu)=S(\mu)$, if $\lambda\left(e_{0}^{+}\right)=-\lambda\left(e_{0}^{\prime}\right) / 16$ and $\mu\left(e_{0}^{+}\right)=-\mu\left(e_{0}^{\prime}\right) / 16$. Every homomorphism $\phi: M(V(\lambda)) \rightarrow M(V(\mu))$ is injective or zero. 
Proof. Let $\phi: M(V(\lambda)) \rightarrow M(V(\mu))$ be a nonzero homomorphism. Then $\phi\left(v_{\lambda}\right)=Y v_{\mu}, Y \neq 0, Y \in U\left(\mathrm{n}^{-}\right) \oplus U\left(\mathrm{n}^{-}\right) e_{0}^{-}$if $V(\lambda)=R(\lambda), V(\mu)=R(\mu)$ [respectively $Y \in U\left(\mathrm{n}^{-}\right)$if $V(\lambda)=\mathbb{C}(\lambda), \quad V(\mu)=\mathbb{C}(\mu), \quad$ or $\left.\quad V(\lambda)=S(\lambda), \quad V(\mu)=S(\mu)\right]$. If $v \in M(V(\lambda)) \backslash\{0\}$, say $v=X v_{\lambda}, \quad X \neq 0, \quad X \in U\left(\mathrm{n}^{-}\right) \oplus U\left(\mathrm{n}^{-}\right) e_{0}^{-}$if $V(\lambda)=R(\lambda)$, $V(\mu)=R(\mu) \quad$ [respectively $X \in U\left(\mathrm{n}^{-}\right)$if $V(\lambda)=\mathbb{C}(\lambda), \quad V(\mu)=\mathbb{C}(\mu)$, or $V(\lambda)$ $=S(\lambda), \quad V(\mu)=S(\mu)]$, then $X Y \neq 0$ by Proposition 3.1. Now, $M(R(\mu)) \simeq\left(U\left(\mathrm{n}^{-}\right) \oplus U\left(\mathrm{n}^{-}\right) e_{0}^{-}\right) \otimes_{\mathbb{C}} \mathbb{C}_{\mu}, \quad M(\mathbb{C}(\mu))=U\left(\mathrm{n}^{-}\right) \otimes_{\mathbb{C}} \mathbb{C}$ and $M(S(\mu))$ $=U\left(\mathrm{n}^{-}\right) \otimes_{\mathbb{C}} \mathbb{C}$, hence $X Y v \neq 0$. Q.E.D.

We now define the contravariant form on $M(V(\lambda))$ for $V(\lambda)=\mathbb{C}(\lambda), R(\lambda)$, or $S(\lambda)$ if $\lambda\left(e_{0}^{+}\right)=-\lambda\left(e_{0}^{\prime}\right) / 16$.

We set $\sigma\left(e_{i}^{+}\right)=e_{-i}^{+}, \sigma\left(e_{i}^{-}\right)=e_{-i}^{-}$and $\sigma\left(e_{0}^{\prime}\right)=e_{0}^{\prime}$. Clearly,

$$
\begin{gathered}
\sigma\left(\left[e_{i}^{+}, e_{j}^{+}\right]\right)=\left[\sigma\left(e_{j}^{+}\right), \sigma\left(e_{i}^{+}\right)\right], \\
\sigma\left(\left[e_{i}^{-}, e_{j}^{-}\right]\right)=\left[\sigma\left(e_{j}^{-}\right), \sigma\left(e_{i}^{-}\right)\right], \\
\sigma\left(\left[e_{i}^{+}, e_{j}^{-}\right]\right)=\left[\sigma\left(e_{j}^{-}\right), \sigma\left(e_{i}^{+}\right)\right], \\
\sigma\left(\left[\mathfrak{g}, e_{0}^{\prime}\right)\right]=0=\left[\sigma\left(e_{0}^{\prime}\right), \sigma(\mathfrak{g})\right] .
\end{gathered}
$$

This implies that $\sigma$ extends to a $\mathbb{Z}_{2}$-graded linear bijection from $\mathfrak{g}$ to $\mathfrak{q}$ such that $\sigma^{2}=$ identity and $\sigma([X, Y])=[\sigma(Y), \sigma(X)]$, for all $X, Y \in \mathfrak{q}$. By the universal property of $U(\mathfrak{g}), \sigma$ therefore extends to a $\mathbb{Z}_{2}$-graded linear bijection from $U(\mathfrak{g})$ to $U(\mathfrak{q})$ such that $\sigma^{2}=$ identity and $\sigma(X Y)=\sigma(Y) \sigma(X)$, for all $X, Y \in U(\mathfrak{g})$. From the decomposition $\mathfrak{g}=\mathfrak{n}^{-} \oplus \mathfrak{g}^{0} \oplus \mathfrak{n}$ and Corollary 2.5, one sees that $U(\mathfrak{g})$ $=U\left(\mathfrak{g}^{0}\right) \oplus\left(\mathrm{n}^{-} U(\mathfrak{g})+U(\mathfrak{g}) \mathfrak{n}\right)$. Let $\phi: U(\mathfrak{g}) \in U\left(\mathfrak{g}^{0}\right)$ be the projection on the first factor, and let $\Pi_{\overline{0}}: U\left(\mathfrak{g}^{0}\right) \rightarrow\left(U\left(\mathfrak{g}^{0}\right)\right)_{\overline{0}}$ be the projection on the even component of $U\left(\mathfrak{g}^{0}\right)$. Let $\beta=\Pi_{\overline{0}} \circ \phi: U(\mathfrak{g}) \rightarrow U\left(\mathfrak{g}^{0}\right)_{0}$. For $X, Y \in U(\mathfrak{g}), \lambda \in \mathfrak{h}^{*}$, we set

$$
\left(X v_{\lambda}, Y v_{\lambda}\right)_{\lambda}=(\lambda \circ \beta)(\sigma(X) Y) \text {, }
$$

where we have extended $\lambda \in \mathfrak{h}^{*}$ canonically to an algebra homomorphism $\lambda: U\left(\mathfrak{g}^{0}\right)_{0} \rightarrow \mathbb{C}$, and $v_{\lambda} \in M(V(\lambda)), V(\lambda)=\mathbb{C}(\lambda), R(\lambda)$, or $S(\lambda)$, if $\lambda\left(e_{0}^{+}\right)=-\lambda\left(e_{0}^{\prime}\right) / 16$.

Proposition 3.14. (a) ( , $)_{\lambda}$ defines a symmetric, bilinear form on $M(V(\lambda))$ such that

$$
(X v, w)_{\lambda}=(v, \sigma(X) w)_{\lambda} \quad \text { for all } \quad X \in U(\mathfrak{g}), v, w \in M(V(\lambda)),
$$

(b) $\left(M(V(\lambda))_{\gamma_{1}}^{\mu_{1}}, M(V(\lambda))_{\gamma_{2}}^{\mu_{2}}\right)_{\lambda}=0$ if $\mu_{1} \neq \mu_{2}$ or $\gamma_{2} \neq \gamma_{2}, \mu_{1}, \mu_{2} \in \mathfrak{h}^{*}, \gamma_{1}, \gamma_{2} \in \mathbb{Z}_{2}$;

(c) The radical of $(,)_{\lambda}$, $\operatorname{Rad}(,)_{\lambda}$, is the maximal proper submodule of $M(V(\lambda))$;

(d) $(,)_{\lambda}$ is the unique symmetric bilinear form on $M(V(\lambda))$ such that $\left(M(V(\lambda))_{\overline{0}}, M(V(\lambda))_{\overline{1}}\right)_{\lambda}=0,\left(v_{\lambda}, v_{\lambda}\right)_{\lambda}=1$ and such that $(6)$ is satisfied.

Proof. (a) If $X v_{\lambda}=0$, then $X$ is in the left ideal of $U(\mathfrak{g})$ generated by $\pi$ and $\{H-\lambda(H) \mid H \in \mathfrak{h}\}$, if $V(\lambda)=\mathbb{C}(\lambda)$ or $R(\lambda)$ [respectively generated by $\mathfrak{n}, \mathbb{C} e_{0}^{-}$and $\{H-\lambda(H) \mid H \in \mathfrak{h}\}$ if $\left.V(\lambda)=S(\lambda), \lambda\left(e_{0}^{+}\right)=-\lambda\left(e_{0}^{\prime}\right) / 16\right]$. Therefore $\left(X v_{\lambda}, U(\mathfrak{g}) v_{\lambda}\right)_{\lambda}=0$. Now,

$$
\left(X v_{\lambda}, Y v_{\lambda}\right)_{\lambda}=(\lambda \circ \beta)(\sigma(X) Y)=(\lambda \circ \beta)(\sigma(\sigma(X) Y))=(\lambda \circ \beta)(\sigma(Y) X)=\left(Y v_{\lambda}, X v_{\lambda}\right)_{\lambda},
$$

hence $(,)_{\lambda}$ is symmetric. Also,

$$
\left(Z X v_{\lambda}, Y v_{\lambda}\right)_{\lambda}=(\lambda \circ \beta)(\sigma(Z X) Y)=(\lambda \circ \beta)(\sigma(X) \sigma(Z) Y)=\left(X v_{\lambda}, \sigma(Z) Y v_{\lambda}\right)_{\lambda} .
$$


(b) $U(\mathfrak{g})_{\bar{i}} U(\mathfrak{g})_{\bar{j}} \subset U(\mathfrak{g})_{\bar{i}+\bar{j}}, \sigma\left(U(\mathfrak{g})_{\bar{i}}\right) \subset U(\mathfrak{g})_{\bar{i}}, i=0,1$, and $\beta\left(U(\mathfrak{g})_{\overline{1}}\right)=(0)$. Hence, $\beta(\sigma(X) Y)=0 \quad$ if $\quad X \in U(\mathfrak{g})_{i}, \quad Y \in U(\mathfrak{g})_{1-i}, \quad i=0,1$. Let now $v_{1} \in M(V(\lambda))^{\mu_{1}}$, $v_{2} \in M(V(\lambda))^{\mu_{2}}$ and $\mu_{1} \neq \mu_{2}$. Then $\left(H v_{1}, v_{2}\right)_{\lambda}=\mu_{1}(H)\left(v_{1}, v_{2}\right)_{\lambda}$ and $\left(v_{1}, H v_{2}\right)$ $=\mu_{2}(H)\left(v_{1}, v_{2}\right)$ for all $H \in \mathfrak{h}$. By $(6)\left(\mu_{1}(H)-\mu_{2}(H)\right)\left(v_{1}, v_{2}\right)_{\lambda}=0$, hence $\left(v_{1}, v_{2}\right)_{\lambda}=0$.

(c) Let $v \in \operatorname{Rad}(,)_{\lambda}$. Then $\left(X v, U(\mathfrak{g}) v_{\lambda}\right)_{\lambda}=\left(v, \sigma(X) U(\mathfrak{g}) v_{\lambda}\right)_{\lambda}=0$ for all $X \in U(\mathrm{~g})$. Hence, $\operatorname{Rad}(,)_{\lambda}$ is a proper submodule of $M(V(\lambda))$, since $\left(v_{\lambda}, v_{\lambda}\right)_{\lambda}=1$. Therefore $\operatorname{Rad}(,)_{\lambda} \subset$ largest proper submodule of $M(V(\lambda))$. Conversely, let $v$ be in the largest proper submodule of $M(V(\lambda))$. Then $v \in\left(\bigoplus_{n>0} M(V(\lambda))^{\lambda-n \delta}\right) \oplus M(V(\lambda)) \frac{\lambda}{1}$, and so, by (b), $\left(v, v_{\lambda}\right)_{\lambda}=0$. Now, $\left(v, U(\mathrm{~g}) v_{\lambda}\right)=\left(\sigma(U(\mathrm{~g})) v, v_{\lambda}\right)_{\lambda}=0$ and $v \in \operatorname{Rad}(,)_{\lambda}$.

(d) Let $\langle,\rangle_{\lambda}$ be a symmetric bilinear form on $M(V(\lambda))$ such that $\left(M(V(\lambda))_{\overline{0}}, M(V(\lambda))_{\overline{1}}\right)=0$ and such that $\langle X v, w\rangle_{\lambda}=\langle v, \sigma(X) w\rangle_{\lambda}$ for all $X \in U(\mathrm{~g})$, $v, w \in M(V(\lambda))$. Then $\left\langle X v_{\lambda}, Y v_{\lambda}\right\rangle_{\lambda}=\left\langle v_{\lambda}, \sigma(X) Y v_{\lambda}\right\rangle_{\lambda}$. By (b), $\left\langle v_{\lambda}, \sigma(X) Y v_{\lambda}\right\rangle_{\lambda}$ $=(\lambda \circ \beta)(\sigma(X) Y)\left\langle v_{\lambda}, v_{\lambda}\right\rangle_{\lambda}$. Q.E.D.

Let $\lambda \in \mathfrak{h}^{*}$ and set $h=\lambda\left(e_{0}^{+}\right), c=\lambda\left(e_{0}^{\prime}\right)$. For $n \in \frac{1}{2} \mathbb{Z}_{+}$and $\gamma \in \mathbb{Z}_{2}$, we denote by $(,)_{h, c, n}\left[\right.$ respectively $\left.(,)_{h, c, n, \gamma}\right]$ or by $(,)_{\lambda, n}\left[\right.$ respectively $\left.(,)_{\lambda, n, \gamma}\right]$ the restriction of $(,)_{\lambda}$ to $M(V(\lambda))^{\lambda-n \delta}\left[\right.$ respectively $\left.M(V(\lambda))^{\lambda-n \delta}\right]$, where $V(\lambda)=\mathbb{C}(\lambda)$ or $R(\lambda)$. We now state the formulas for the determinant of $(,)_{h, c, n, \gamma}$ (we omit the subscript $\gamma$ if $\mathfrak{g}=\widehat{n s}$ ). The formula for the Neveu-Schwarz algebra was conjectured by Kac [14], and the one for the Ramond algebra was conjectured by D. Friedan, Z. Qiu, and S. H. Shenker $[7,8]$. Let $r, s \in \frac{1}{2} \mathbb{N}, r \leqq s$. Set, for $\mathfrak{g}=\widehat{\mathfrak{r} \mathfrak{s}}$

$$
h_{r, s}^{ \pm}(c)=-\frac{1}{8}\left[\left(r^{2}+s^{2}\right)(5-c) \pm \sqrt{c^{2}-10 c+9}\left(r^{2}-s^{2}\right)-8 r s-\frac{1}{2}+\frac{1}{2} c\right],
$$

and, for $\mathfrak{g}=\hat{\mathfrak{r}}$

$$
h_{r, s}^{ \pm}(c)=-\frac{1}{8}\left[\left(r^{2}+s^{2}\right)(5-c) \pm \sqrt{c^{2}-10 c+9}\left(r^{2}-s^{2}\right)-8 r s-\frac{1}{2}+\frac{1}{2} c\right]-\frac{1}{16} .
$$

Also, set, for $r \neq s, \psi_{r, s}(h, c)=\left(h-h_{r, s}^{+}(c)\right)\left(h-h_{r, s}^{-}(c)\right)$, and, for $\mathfrak{g}=\hat{\mathfrak{r} s}, \psi_{r, r}(h, c)=h$ $+\frac{1}{16}(1-c)\left(4 r^{2}-1\right)$. Let $\hat{p}(i)$ be as in Proposition 3.6. Then, if $\mathfrak{g}=\hat{\mathfrak{r}} \hat{\mathfrak{s}}$,

and, for $\mathfrak{g}=\hat{\mathfrak{x}}$,

$$
\operatorname{det}(,)_{h, c, n}=\prod_{2 r \leqq \leqq n, r-s \in \mathbb{Z}} \psi_{r, s}(h, c)^{\hat{p}(n-2 r s)},[14]
$$

$$
\operatorname{det}(,)_{h, c, n, \gamma}=\left(h+\frac{c}{16}\right)^{\frac{1}{2} \hat{p}(n)} \prod_{2 r s \leqq n, r-s \in \frac{1}{2}+\mathbb{Z}} \psi_{r, s}(h, c)^{\hat{p}(n-2 r s)}
$$

if $n \geqq 0$, and $\operatorname{det}(,)_{h, c, 0, \overline{0}}=1, \operatorname{det}(,)_{h, c, 0, \overline{1}}=h+\frac{c}{16}[7,8]$.

Note. The nonzero constant depending on the choice of a basis of $M(V(\lambda))$ has been omitted in (8), (9).

\section{The Representations $\mathscr{K}^{\check{\zeta}}$ and the Proof of the Determinant Formulas}

We retain the notation of Sect. 3. In this section we prove the determinant formulas for the restriction of $(,)_{\lambda}$ to $M(V(\lambda))_{\gamma}^{\mu}$ (see Sect. 3). The main ingredients are the 
construction of the representations $\mathscr{K}^{\xi, \omega}$ of $\mathfrak{g}$ (Theorem 4.2), which generalize the construction of [11], and the vertex operator.

Following [6] let $\mathrm{t}$ be a one-dimensional space, $\mathfrak{t}=\mathbb{C} T$, and let $\langle$, $\rangle$ be a symmetric, bilinear form on $\mathrm{t}$ such that $\langle T, T\rangle=1$. Let $\hat{\mathfrak{t}}=\mathrm{t} \otimes \mathbb{C}\left[t, t^{-1}\right] \oplus \mathbb{C} z$, and let

$$
\begin{gathered}
{[X(m), Y(n)]=\langle X, Y\rangle m \delta_{m+n, 0} z,} \\
{[\hat{\mathrm{t}}, z]=0,}
\end{gathered}
$$

where we have set $X(m)=X \otimes t^{m}$ for all $x \in \mathrm{t}, m \in \mathbb{Z}$. We let

$$
\begin{gathered}
D\left(X \otimes t^{n}\right)=n, \quad X \in \mathrm{t}, n \in \mathbb{Z}, \\
D(z)=0 .
\end{gathered}
$$

We note that $\hat{\mathfrak{t}}_{*}=\left(\underset{n \in \mathbb{Z} \backslash\{0\}}{\bigoplus} \mathfrak{t} \otimes \mathbb{C} t^{n}\right) \oplus \mathbb{C} z$ is a $\mathbb{Z}$-graded Heisenberg algebra with the $\mathbb{Z}$-grading given by the degree map $D$. Let $\hat{\mathfrak{t}}^{-}=\bigoplus_{n<0} \mathrm{t} \otimes \mathbb{C} t^{n} C \hat{\mathfrak{t}}_{*}$, and $\mathfrak{u}=\left(\bigoplus_{n>0} \mathfrak{t} \otimes \mathbb{C} t^{n}\right) \oplus \mathbb{C} z \subset \hat{\mathfrak{t}}_{*}$. Let $\mathbb{C}(1)$ denote the one-dimensional u-module such that $\left(X \otimes t^{n}\right) 1=0$ if $X \in \mathrm{t}, n>0$, and $z 1=1$, and form the left $U\left(\hat{\mathfrak{t}}_{*}\right)$-module: $\operatorname{Ind}_{\mathfrak{u}}^{\hat{\mathfrak{t}}_{*}} \mathbb{C}(1)$. Note that $S\left(\hat{\mathfrak{t}}^{-}\right)$is linearly isomorphic with $\operatorname{Ind}_{\mathfrak{u}} \hat{\mathrm{t}}_{*} \mathbb{C}(1)$. Using this isomorphism we can define a $\hat{\mathfrak{t}}_{*}$-module action on $S\left(\hat{\mathfrak{t}}^{-}\right)$and identify $S\left(\hat{\mathfrak{t}}^{-}\right)$with $\operatorname{Ind}_{\mathfrak{u}}^{\hat{\mathfrak{t}}_{+}} \mathbb{C}(1)$. Let $\mathbb{C}[\mathrm{t}]$ denote the group algebra of $\mathrm{t}, \mathbb{C}[\mathrm{t}]=\bigoplus_{\omega \in \mathbb{C}} e^{\omega T}$, where $e^{\alpha} e^{\beta}$
$=e^{\alpha+\beta}, \alpha, \beta \in \mathrm{t}$, and let

$$
D e^{\alpha}=-\frac{\langle\alpha, \alpha\rangle}{2}, \quad \alpha \in \mathfrak{t}
$$

Let $V=S\left(\hat{\mathfrak{t}}^{-}\right) \otimes \mathbb{C}[\mathrm{t}]$. We define a representation $\left(\pi_{1}, V\right)$ of $\hat{\mathfrak{t}}$ as follows:

$$
\begin{gathered}
\pi_{1}(X)=X \otimes 1, \quad X \in \hat{\mathfrak{t}}_{*}, \\
\pi_{1}(X)\left(v \otimes e^{\alpha}\right)=v \otimes\langle X, \alpha\rangle e^{\alpha}, X \in \mathfrak{t}, v \in S\left(\hat{\mathfrak{t}}^{-}\right), \quad \alpha \in \mathfrak{t}, \\
\pi_{1}(z)=\text { identity. }
\end{gathered}
$$

Let

$$
\pi_{2}\left(e^{\alpha}\right)\left(v \otimes e^{\beta}\right)=v \otimes e^{\alpha+\beta}, \quad \alpha, \beta \in \mathrm{t}, v \in S\left(\hat{\mathfrak{t}}^{-}\right) .
$$

The pair $(\hat{\mathrm{t}}, \mathbb{C}[\mathrm{t}])$ is called a Heisenberg system $([6])$ and $\left(\pi_{1}, \pi_{2}\right)$ is an irreducible representation of $(\hat{\mathfrak{t}}, \mathbb{C}[\mathrm{t}])([6])$.

Next we describe the spin representation of $[4,17]$. We denote by $Z$ the set $\mathbb{Z}+\frac{1}{2}$ or the set $\mathbb{Z}$. Let $U=\bigoplus_{m \in \mathbb{Z}} \mathbb{C} a(m), U^{+}=\bigoplus_{m>0, m \in Z} \mathbb{C} a(m)$, and $U^{-}=\bigoplus_{m \leqq 0, m \in Z} \mathbb{C} a(m)$. Let $\langle\rangle:, U \times U \rightarrow \mathbb{C}$ be the symmetric bilinear form satisfying

$$
\langle a(m), a(n)\rangle=\delta_{m+n, 0},
$$

and let $\mathscr{C} \ell(U)$ be the corresponding Clifford algebra. That is, $\mathscr{C} \ell(U)=T(U) / J$, where $T(U)$ is the tensor algebra of $U$ and $J$ is the two-sided ideal of $T(U)$ 
generated by the elements of the form: $v \otimes w+w \otimes v-\langle v, w\rangle$. Let

$$
\operatorname{Da}(m)=m \text {. }
$$

We define, for $X \in U^{-}, Y \in U^{+}$,

$$
\begin{gathered}
L_{X}\left(v_{1} \wedge \ldots \wedge v_{n}\right)=X \wedge v_{1} \wedge \ldots \wedge v_{n} \\
l(Y)\left(v_{1} \wedge \ldots \wedge v_{n}\right)=\sum_{j=1}^{n}(-1)^{j-1}\left\langle Y, v_{j}\right\rangle v_{1} \wedge \ldots \wedge \hat{v}_{j} \wedge \ldots \wedge v_{n}, \\
\varrho(X+Y)=L_{X}+l(Y),
\end{gathered}
$$

$v_{i} \in U^{-}, i=1, \ldots, n . \varrho$ extends to an irreducible representation of $\mathscr{C} \ell(U)$ on $\mathscr{C} \ell\left(U^{-}\right)$ $=\Lambda\left(U^{-}\right)$, also denoted by $\varrho$, called the spin representation corresponding to the polarization $U=U^{+} \oplus U^{-}([4,17])$. Let $W=V \otimes \Lambda\left(U^{-}\right)$. We let $(\hat{\mathfrak{t}}, \mathbb{C}[t])$ act on $W$ by letting it act trivially on $\Lambda\left(U^{-}\right)$, and let $\mathscr{C} \ell(U)$ act on $W$ by letting it act trivially on $V$. We make $W$ into a $\mathbb{Z}_{2}$-graded vector space $W=\bigoplus_{\gamma \in \mathbb{Z}_{2}} W_{\gamma}$ by setting $\operatorname{deg} v=\overline{0}$
for all $v \in V$ and $\operatorname{deg} u=\overline{1}$ for all $u \in U$.

From now on we omit the representation symbols and indicate the action of an element of $(\hat{\mathrm{t}}, \mathbb{C}[t])$ or $\mathscr{C} \ell(U)$ on $W$ by the element itself.

The ordered products: :T(m) T(n): and $: a(m) a(n)$ : of the operators $T(m)$ and $T(n)$, and $a(m)$ and $a(n)$, respectively, are defined as follows:

$$
\begin{gathered}
: T(m) T(n):= \begin{cases}T(m) T(n) & \text { if } m<n, \\
\frac{1}{2}[T(m) T(n)+T(n) T(m)] & \text { if } m=n, \\
T(n) T(m) & \text { if } m>n,\end{cases} \\
: a(m) a(n):=\left\{\begin{array}{lll}
a(m) a(n) & \text { if } m<n, \\
\frac{1}{2}[a(m) a(n)-a(n) a(m)] & \text { if } m=n, \\
-a(n) a(m) & \text { if } m>n .
\end{array}\right.
\end{gathered}
$$

We now define the operators that give a special case of the representations of $g$ that will be constructed. From now on, $Z=\mathbb{Z}+\frac{1}{2}$ if $\mathfrak{g}=\hat{\mathfrak{r} s}$ and $Z=\mathbb{Z}$ if $\mathfrak{g}=\hat{\mathfrak{r}}$.

Let, for $n \in \mathbb{Z}$,

$$
d_{n}^{+}=-\frac{1}{2} \sum_{k \in \mathbb{Z}}: T(k) T(n-k):+\frac{1}{2} \sum_{k \in \mathbb{Z}+\frac{1}{2}} k: a(k) a(n-k):
$$

if $\mathfrak{g}=\hat{\mathfrak{s}}$

$$
d_{n}^{+}=-\frac{1}{2} \sum_{k \in \mathbb{Z}}: T(k) T(n-k):+\frac{1}{2} \sum_{k \in \mathbb{Z}} k: a(k) a(n-k):-\frac{1}{16} \delta_{n, 0}
$$

if $\mathfrak{g}=\hat{\mathfrak{x}}$, and, for $n \in Z$,

$$
d_{n}^{-}=\sum_{k \in \mathbb{Z}} T(k) \otimes a(n-k) .
$$

(We sometimes drop the superscripts if $\mathfrak{g}=\hat{\mathrm{rs}}$.)

The degree map $D$ given by (3), (4), (5), and (11) extends uniquely to a degree derivation on $W$ which we also denote by $D$. 
If $A, B, C$ are operators we have the following obvious identities: $[A B, C]$ $=[A, C] B+A[B, C], \quad[A B, C]=-[A, C]_{+} B+A[B, C]_{+} \quad$ and $\quad[A B, C]_{+}$ $=-[A, C] B+A[B, C]_{+}$, where $[A, B]_{+}$is the anticommutator $A B+B A$.

Let $d_{n}^{+, 0}=-\frac{1}{2} \sum_{k \in \mathbb{Z}}: T(k) T(n-k):, n \in \mathbb{Z}$. Then one has:

$$
\begin{gathered}
{\left[d_{m}^{+, 0}, T(n)\right]=n T(m+n), m, n \in \mathbb{Z} \quad([6], \text { see also [12]) }} \\
{\left[d_{m}^{+, 0}, d_{n}^{+, 0}\right]=(n, m) d_{m+n}^{+, 0}+\frac{m^{3}-m}{12} \delta_{m+n, 0}, m, n \in \mathbb{Z}}
\end{gathered}
$$

([6], see also [12]). The relations of the next lemma were also known $([5]) \cdot{ }^{4} \mathrm{We}$ give their proof for completeness.

Lemma 4.1. Let $\quad d_{n}^{+, 1}=\frac{1}{2} \sum_{k \in \mathbb{Z}+\frac{1}{2}} k: a(k) a(n-k): \quad$ if $\quad \mathfrak{g}=\hat{\mathfrak{T} S} \quad$ and $\quad d_{n}^{+, 1}$

$$
\begin{aligned}
& =\frac{1}{2} \sum_{k \in \mathbb{Z}} k: a(k) a(n-k):-\frac{1}{16} \delta_{n} \text { if } \mathrm{g}=\hat{\mathrm{r}} \text {. Then } \\
& \quad \text { (i) }\left[d_{m}^{+, 1}, a(n)\right]=\left(n+\frac{1}{2} m\right) a(m+n), m \in \mathbb{Z}, n \in Z ; \\
& \quad \text { (ii) }\left[d_{m}^{+, 1}, d_{n}^{+, 1}\right]=(n-m) d_{m+n}^{+, 1}+\frac{m^{3}-m}{24} \delta_{m+n, 0}, m, n \in \mathbb{Z} .
\end{aligned}
$$

Proof. (i) $\quad\left[d_{m}^{+, 1}, a(n)\right]=\frac{1}{2} \sum_{k \in Z} k[a(k) a(m-k), a(n)]$, by (16). Assume that $m \neq-2 n$. Then $\left[d_{m}^{+, 1}, a(n)\right]=\frac{1}{2}[-n a(-n) a(m+n)+(m+n) a(m+n) a(-n), a(n)]$ $=\frac{1}{2}(m+2 n) a(m+n)$, by (10). If $m=-2 n$, then, $\left[d_{m}^{+, 2}, a(n)\right]$ $=\frac{1}{2}[-n a(-n) a(-n), a(n)]=0$;

(ii) $\left[\left[d_{m}^{+, 1}, d_{n}^{+, 1}\right], a(k)\right]=\left[d_{m}^{+, 1},\left(k+\frac{1}{2} n\right) a(k+n)\right]+\left[\left(k+\frac{1}{2} m\right) a(k+m), d_{n}^{+, 1}\right]$ $=\left(k+\frac{1}{2} n\right)\left(k+n+\frac{1}{2} m\right) a(k+m+n)-\left(k+\frac{1}{2} m\right)\left(k+m+\frac{1}{2} n\right) a(k+m+n)=(n-m)$ $\left(k+\frac{1}{2}(m+n)\right) a(m+n+k)=\left[(n-m) d_{m+n}^{+, 1}, a(k)\right]$ for all $k \in Z$, by (i). Therefore, $\left[d_{m}^{+, 1}, d_{n}^{+, 1}\right]-(n-m) d_{m+n}^{+, 1}$ is constant, by the irreducibility of $\Lambda\left(U^{-}\right)$. Since $\left[d_{m}^{+, 1}, d_{n}^{+, 1}\right]-(n-m) d_{m+1}^{+, 1}$ is homogeneous of degree $m+n$ relative to $D$, we conclude that this constant is zero unless $m+n=0$. Now, if $\mathfrak{g}=\hat{\mathfrak{n}} \mathfrak{s}$ $\left[d_{1}^{+, 1}, d_{-1}^{+, 1}\right](1 \otimes 1 \otimes 1)=d_{1}^{+, 1}, d_{-1}^{+, 1}(1 \otimes 1 \otimes 1)=\left(\frac{1}{4} a\left(\frac{1}{2}\right)^{2}\right)\left(-\frac{1}{4} a\left(-\frac{1}{2}\right)^{2}\right)(1 \otimes 1 \otimes 1)$ $=0=-2 d_{0}^{+, 1}(1 \otimes 1 \otimes 1)$. Similarly,

$$
\left[d_{2}^{+, 1}, d_{-2}^{+, 1}\right](1 \otimes 1 \otimes 1)=\frac{1}{4}(1 \otimes 1 \otimes 1)=-4 d_{0}^{+, 1}(1 \otimes 1 \otimes 1)
$$

and

$$
\left[d_{1}^{+, 1}, d_{-1}^{+, 1}\right](1 \otimes 1 \otimes 1)=\frac{1}{8}(1 \otimes 1 \otimes 1)=-2 d_{0}^{+, 1}(1 \otimes 1 \otimes 1)
$$

and

$$
\left[d_{2}^{+, 1}, d_{-2}^{+, 1}\right](1 \otimes 1 \otimes 1)=\frac{1}{2}(1 \otimes 1 \otimes 1)=\left(-4 d_{0}^{+, 1}+\frac{1}{4}\right)(1 \otimes 1 \otimes 1) \quad \text { if } \quad \mathfrak{g}=\hat{\mathfrak{r}} .
$$

Proceeding by induction, we assume that (ii) has been proved for $n=-m$ and $m \geqq 2$. Now, write $d_{m+1}^{+, 1}=\frac{1}{m-1}\left[d_{1}^{+, 1}, d_{m}^{+, 1}\right]$ and $d_{-m-1}^{+, 1}=\frac{-1}{m-1}\left[d_{-1}^{+, 1}, d_{-m}^{+, 1}\right]$. It is now a simple exercise to see that $\left[d_{m+1}^{+, 1}, d_{-m-1}^{+, 1}\right]=-2(m+1) d_{0}^{+, 1}$ $+\frac{(m+1)^{3}-(m+1)}{24}$, which proves (ii). Q.E.D.

\footnotetext{
${ }^{4}$ Similar calculations were also carried out in [3]
} 
Lemma 4.1, (20) and (21) imply that the formulas (17)-(19) give a representation of $\mathfrak{g}$ on $W$ where the central element acts as the identity operator. To obtain arbitrary central action, we let, for $\xi \in \mathbb{C}$ and $n \in \mathbb{Z}$,

$$
e_{n}^{+}(\xi)=d_{n}^{+}+\xi n T(n)+\frac{\xi^{2}}{2} \delta_{n, 0}
$$

and, for $n \in Z$,

$$
e_{n}^{-}(\xi)=d_{n}^{-}-2 \xi n a(n) .
$$

(We sometimes drop the superscripts if $\mathfrak{g}=\hat{\mathfrak{r}}$.)

We note that $[A \otimes B, C \otimes D]=[A, C] \otimes B D+C A \otimes[B, D]$ and $[A \otimes B$, $C \otimes D]_{+}=[A, C] \otimes B D+C A \otimes[B, D]_{+}$.

Theorem 4.2. Let $\xi \in \mathbb{C}$ and set $\pi_{\xi}\left(e_{n}^{+}\right)=e_{n}^{+}(\xi), n \in \mathbb{Z}, \pi_{\xi}\left(e_{n}^{-}\right)=e_{n}^{-}(\xi), n \in Z$, and $\pi_{\xi}\left(e_{0}^{\prime}\right)=1-8 \xi^{2}$. Then $\pi_{\xi}$ extends to a representation of $\mathfrak{g}$ on $W$.

Proof. (a) $\left[e_{m}^{+}(\xi), e_{n}^{+}(\xi)\right]=\left[d_{m}^{+, 0}, d_{n}^{+, 0}\right]+\xi n\left[d_{m}^{+, 0}, T(n)\right]+\left[d_{m}^{+, 1}, d_{n}^{+, 1}\right]+\xi m$ $\cdot\left[T(m), d_{n}^{+, 0}\right]+\xi^{2} m n[T(m), T(n)]=(n-m) d_{m+n}^{+, 0}+\frac{m^{3}-m}{12} \delta_{m+n, 0}+\xi n^{2} T(m+n)$ $+(n-m) d_{m+n}^{+, 1}+\frac{m^{3}-m}{24} \delta_{m+n, 0}-\xi m^{2} T(m+n)+\xi^{2} m^{2} n \delta_{m+n, 0}$ by (20), (21), and
Lemma 4.1.

Hence, $\quad\left[e_{m}^{+}(\xi), e_{n}^{+}(\xi)\right]=(n-m)\left[d_{m+n}^{+}+\xi(m+n) T(m+n)+\frac{\xi^{2}}{2} \delta_{m+n, 0}\right]$ $+\frac{m^{3}-m}{8} \delta_{m+n, 0}-\xi^{2} m^{3} \delta_{m+n, 0}+m \xi^{2} \delta_{m+n, 0}=(n-m) e_{m+n}^{+}(\xi)$ $+\frac{m^{3}-m}{8} \delta_{m+n, 0}\left(1-8 \xi^{2}\right)$

(b) $\left[e_{m}^{+}(\xi), e_{n}^{-}(\xi)\right]=\left[d_{m}^{+, 0}, d_{n}^{-}\right]+\left[d_{m}^{+, 1}, d_{n}^{-}\right]+\xi m\left[T(m), d_{n}^{-}\right]-2 \xi n\left[d_{m}^{+, 1}, a(n)\right]$ $=\sum_{k \in \mathbb{Z}}\left[\left[d_{m}^{+, 0}, T(k)\right] \otimes a(n-k)+T(k)\left[d_{m}^{+, 1}, a(n-k)\right]\right.$ $+\xi m[T(m), T(k)] \otimes a(n-k)]-2 \xi n\left[d_{m}^{+, 1}, a(n)\right]=\sum_{k \in \mathbb{Z}}[k T(m+k) \otimes a(n-k)$ $\left.+\left(n-k+\frac{1}{2} m\right) T(k) \otimes a(m+n-k)\right]+\xi m^{2} a(m+n)-2 \xi n\left(n+\frac{1}{2} m\right) a(m+n)$ $=\sum_{k \in \mathbb{Z}}\left[\left(k-m+n-k+\frac{1}{2} m\right) T(k) \otimes a(m+n-k)\right]+\xi\left(m^{2}-2 n^{2}-m n\right) a(m+n)$ $=\sum_{k \in \mathbb{Z}}\left(n-\frac{1}{2} m\right) T(k) \otimes a(m+n-k)-2 \xi\left(n-\frac{1}{2} m\right)(m+n) a(m+n)=\left(n-\frac{1}{2},\right) e_{m+n}^{-}(\xi)$, by (20) and Lemma 4.1 .

(c) $\left[e_{m}^{-}(\xi), e_{n}^{-}(\xi)\right]_{+}=\sum_{k, l \in \mathbb{Z}}[T(k) \otimes a(m-k), T(l) \otimes a(n-l)]_{+}$

$-2 \xi n \sum_{k \in \mathbb{Z}}[T(k) \otimes a(m-k), a(n)]_{+}-2 \xi m \sum_{l \in \mathbb{Z}}[a(m), T(l) \otimes a(n-l)]_{+}$ $+4 \xi^{2} m n[a(m), a(n)]_{+}=\sum_{k, l \in \mathbb{Z}}[[T(k), T(l)] \otimes a(m-k) a(n-l)$ $\left.+T(l) T(k) \otimes[a(m-k), a(n-l)]_{+}\right]-2 \xi n \sum_{k \in \mathbb{Z}} T(k) \otimes[a(m-k), a(n)]_{+}$ $-2 \xi m \sum_{l \in \mathbb{Z}} T(l) \otimes[a(m), a(n-l)]_{+}+4 \xi^{2} m n[a(m), a(n)]_{+}=\sum_{k \in \mathbb{Z}}[k a(m-k) a(n+k)$ $+T(m+n-k) T(k)]-2 \xi n T(m+n)-2 \xi m T(m+n)-4 \xi^{2} m^{2} \delta_{m+n, 0}$.

It is clear that $k a(m-k) a(n+k)-k: a(m-k) a(n+k):=0=T(m+n-k) T(k)$ $-: T(m+n-k) T(k)$ : unless $m+n=0$. Assume that $m>0$. If $k>m$, then both 
differences are zero since also $k>0$. If $k<0$, then $k a(m-k) a(n+k)$ $-k: a(m-k) a(n+k):=k \delta_{m+n, 0} \quad$ and $\quad T(m+n-k) T(k)-: T(m+n-k) T(k):$

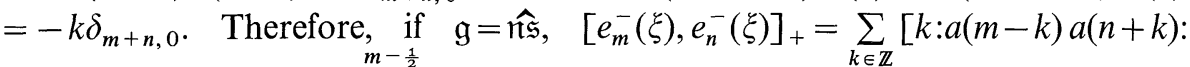
$+: T(m+n-k) T(k):]+\sum_{k=0}^{m-\frac{1}{2}} k \delta_{m+n, 0}-2 \xi(m+n) T(m+n)-4 \xi^{2} m^{2} \delta_{m+n, 0}$ $=m \sum_{k \in \mathbb{Z}+\frac{1}{2}}: a(k) a(m+n-k):-\sum_{k \in \mathbb{Z}+\frac{1}{2}} k: a(k) a(m+n-k):+\sum_{k \in \mathbb{Z}}: T(k) T(m+n-k):$ $+\frac{1}{2}\left(m^{2}-\frac{1}{4}\right) \delta_{m+n, 0}-2 \xi(m+n) T(m+n)-4 \xi^{2} m^{2} \delta_{m+n, 0}=-2 e_{m+n}^{+}(\xi)$ $+\frac{1}{2}\left(m^{2}-\frac{1}{4}\right) \delta_{m+n, 0}\left(1-8 \xi^{2}\right)$. If $\mathfrak{g}=\hat{\mathfrak{r}}, \quad\left[e_{m}^{-}(\xi), e_{n}^{-}(\xi)\right]_{+}=\sum_{k \in \mathbb{Z}}[k: a(m-k) a(n+k):$ $+: T(m+n-k) T(k):]+\sum_{k=0}^{m-1} k \delta_{m+n, 0}+\frac{m}{2} \delta_{m+n, 0}-2 \xi(m+n) T(m+n)$ $-4 \xi^{2} m^{2} \delta_{m+n, 0}=m \sum_{k \in \mathbb{Z}}: a(k) a(m+n-k):-\sum_{k \in \mathbb{Z}} k: a(k) a(m+n-k):$

$+\sum_{k \in \mathbb{Z}}: T(k) T(m+n-k):+\frac{m^{2}}{2} \delta_{m+n, 0}-2 \xi(m+n) T(m+n)-4 \xi^{2} m^{2} \delta_{m+n, 0}$ $=-2 e_{m+n}^{+}(\xi)+\frac{1}{2}\left(m^{2}-\frac{1}{4}\right) \delta_{m+n, 0}\left(1-8 \xi^{2}\right)$. Similarly for $m \leqq 0$. Q.E.D.

For $\omega \in \mathbb{C}$, let $\mathscr{K}^{\xi, \omega}=S\left(\hat{\mathfrak{t}}^{-}\right) \otimes \mathbb{C} e^{\omega T} \otimes \Lambda\left(U^{-}\right)$. Then

$$
W=\bigoplus_{\omega \in \mathbb{C}} \mathscr{K}^{\xi, \omega}
$$

as representations of $\mathfrak{g}$. Let $\mathfrak{h}, \mathfrak{n} \subset \mathfrak{g}$ be as in Sect. 3. For $\mu \in \mathfrak{h}^{*}$ we denote by $\left(\mathscr{K}^{\xi, \omega}\right)^{\mu}$ the $\mu$-weightspace of $\mathscr{K}^{\xi, \omega}$ relative to $\mathfrak{h}$. Let $\left(\mathscr{K}^{\xi, \omega}\right)_{\gamma}^{\mu}=\left(\mathscr{K}^{\xi, \omega}\right)^{\mu} \cap\left(\mathscr{K}^{\xi, \omega}\right)_{\gamma}, \gamma \in \mathbb{Z}_{2}$.

Proposition 4.3. Let $\xi, \omega \in \mathbb{C}$. Then

(i) $\mathscr{K}^{\xi, \omega}=\bigoplus_{\mu \in \mathfrak{h}^{*}}\left(\mathscr{K}^{\mu, \omega}\right)^{\mu}$;

(ii) $\left(\mathscr{K}^{\xi, \omega}\right)^{\mu} \neq 0$ only if $\mu\left(e_{0}^{\prime}\right)=1-8 \xi^{2}$ and $\mu\left(e_{0}^{+}\right)=\frac{\xi^{2}-\omega^{2}}{2}-n$ for some $n \in \frac{1}{2} \mathbb{Z}_{+}$, if $\mathfrak{g}=\hat{\mathfrak{r}} \mathfrak{s}$, and $\mu\left(e_{0}^{+}\right)=\frac{\xi^{2}-\omega^{2}}{2}-\frac{1}{16}-n$ for some $n \in \mathbb{Z}_{+}$, if $\mathfrak{g}=\hat{\mathfrak{r}}$;

(iii) $\operatorname{dim}\left(\mathscr{K}^{\xi, \omega}\right)_{\gamma}^{\mu}=\hat{p}(n)$ for $\mu$ as in (ii) and $\gamma \in \mathbb{Z}_{2}$, where $\hat{p}$ is defined in Proposition 3.5;

(iv) $1 \otimes e^{\omega T} \otimes 1$ is an $\mathrm{n}$-invariant vector in $\mathscr{K}^{\xi, \omega}$.

Proof. Let $k_{1}, \ldots, k_{r} \in \mathbb{N}, l_{1}, \ldots, l_{s} \in \frac{1}{2} \mathbb{N}$, and let $n=\sum_{i=1}^{r} k_{i}+\sum_{j=1}^{s} l_{j}$.

Using (20) and Lemma 4.1 (i) we obtain

$$
\begin{aligned}
e_{0}^{+}(\xi) & \left(T\left(-k_{1}\right) \ldots T\left(-k_{r}\right) \otimes e^{\omega T} \otimes a\left(-l_{1}\right) \ldots a\left(-l_{s}\right)\right. \\
\quad= & T\left(-k_{1}\right) \ldots T\left(-k_{r}\right) a\left(-l_{1}\right) \ldots a\left(-l_{s}\right)\left(e_{0}^{+}(\xi)-\sum_{i=1}^{r} k_{i}-\sum_{j=1}^{s} l_{j}\right)\left(1 \otimes e^{\omega T} \otimes 1\right) \\
\quad & \left(\frac{\xi^{2}-\omega^{2}}{2}-n\right) T\left(-k_{1}\right) \ldots T\left(-k_{r}\right) \otimes e^{\omega T} \otimes a\left(-l_{1}\right) \ldots a\left(-l_{s}\right),
\end{aligned}
$$

if $\mathfrak{g}=\hat{\mathfrak{r}} \hat{\mathfrak{s}}$. Similarly, for $\mathfrak{g}=\hat{\mathfrak{r}}$. This proves (i) and (ii), and (iii) follows from (ii). By Theorem $4.2, e_{0}^{+}$acts on $e_{m}^{ \pm}\left(1 \otimes e^{\omega T} \otimes 1\right)$ by $\frac{\xi^{2}-\omega^{2}}{2}+m$ if $\mathfrak{g}=\widehat{\pi} \mathfrak{s}$, and by $\frac{\xi^{2}-\omega^{2}}{2}$ $-\frac{1}{16}+m$ if $\mathfrak{g}=\hat{\mathfrak{x}}$. (iv) now follows from (ii). Q.E.D. 
Let $\zeta$ be a formal variable and let $\alpha \in \mathbb{C}$. Let

$$
\begin{aligned}
X(\alpha, \zeta)= & \exp \left(\sum_{n \in \mathbb{N}} \frac{\alpha T(-n)}{n} \zeta^{-n}\right) \exp \left(-\sum_{n \in \mathbb{N}} \frac{\alpha T(n)}{n} \zeta^{n}\right) \\
& \otimes e^{\alpha T \zeta^{-\alpha T-\frac{\alpha^{2}}{2}} \otimes 1,}
\end{aligned}
$$

where we set

$$
\zeta^{-\alpha T-\frac{\alpha^{2}}{2}}\left(e^{\omega T}\right)=e^{\omega T} \zeta^{-\alpha w-\frac{\alpha^{2}}{2}},[6] .
$$

We define $X_{\alpha}(b): W \rightarrow W$ by

$$
X(\alpha, \zeta)=\sum_{b \in \mathbb{C}} X_{\alpha}(b) \zeta^{b} .
$$

Then

$$
\begin{gathered}
{\left[T(m), X_{\alpha}(b)\right]=\alpha X_{\alpha}(m+b), m \in \mathbb{Z}, \alpha, b \in \mathbb{C}[6]} \\
{\left[d_{m}^{+, 0}, X_{\alpha}(b)\right]=\left(m+b-\frac{\alpha^{2}}{2} m\right) X_{\alpha}(m+b), m \in \mathbb{Z}, \alpha, b \in \mathbb{C},[6] .}
\end{gathered}
$$

The vertex operator is given by

$$
Y_{\alpha}(b)=\sum_{k \in Z} X_{\alpha}(b-k) \otimes a(k),[5] .
$$

Theorem 4.4. Let $\alpha \in \mathbb{C} \backslash\{0\}$ and let $Y_{\alpha}(b)$ be as in (28). $Y_{\alpha}(0)$ is a $\mathfrak{g}$-homomorphism of $\left(\pi^{\xi}, W\right)$ if and only if $\xi=\frac{\alpha^{2}-1}{2 \alpha} .5$

Proof. $\left[e_{m}^{-}(\xi), Y_{\alpha}(b)\right]_{+}=\left[\sum_{k \in \mathbb{Z}} T(k) \otimes a(m-k)-2 \xi m a(m), \sum_{l \in \mathbb{Z}+\frac{1}{2}} X_{\alpha}(b-l) \otimes a(l)\right]_{+}$ $=\sum_{k, l}\left[\left[T(k), X_{\alpha}(b-l)\right] \otimes a(m-k) a(l)+X_{\alpha}(b-l) T(k) \otimes[a(m-k), a(l)]\right]$

$-2 \xi m X_{\alpha}(b+m)=\sum_{k, l}\left[\alpha X_{\alpha}(k+b-l) \otimes a(m-k) a(l)\right.$

$\left.+X_{\alpha}(b+m-k) T(k) \otimes \delta_{m-k+l, 0}\right]-2 \xi m X_{\alpha}(b+m)$ by (26). This implies that $\left[e_{m}^{-}(\xi), Y_{\alpha}(b)\right]_{+}=\sum_{k \in \mathbb{Z}}\left[\alpha X_{\alpha}(b+m) \otimes a(m-k) a(k-m)+X_{\alpha}(b+m-k) T(k)\right]$

$-2 \xi m X_{\alpha}(b+m)$. We define : $X_{\alpha}(b+m-k) T(k)$ : to be $X_{\alpha}(b+m-k) T(k)$ if $k>0$, $\frac{1}{2}\left[X_{\alpha}(b+m-k) T(k)+T(k) X_{\alpha}(b+m-k)\right]$ if $k=0$, and $T(k) X_{\alpha}(b+m-k)$ if $k<0$. Assume that $m>0$. If $k>m$; then $a(m-k) a(k-m)-: a(m-k) a(k-m):=0$ $=X_{\alpha}(b+m-k) T(k)-: X_{\alpha}(b+m-k) T(k)$. If $k<0$, then

$$
\alpha X_{\alpha}(b+m) \otimes[a(m-k) a(k-m)-: a(m-k) a(k-m):]=\alpha X_{\alpha}(b+m),
$$

and $X_{\alpha}(b+m-k) T(k)-: X_{\alpha}(b+m-k) T(k):=-\alpha X_{\alpha}(b+m)$ by (26). Hence, $\left[e_{m}^{-}(\xi), Y_{\alpha}(b)\right]_{+}=\sum_{k \in \mathbb{Z}}\left[\alpha X_{\alpha}(b+m) \otimes: a(m-k) a(k-m):+: X_{\alpha}(b+m-k) T(k):\right]$ $+\sum_{k=1}^{m-\frac{1}{2}} \alpha X_{\alpha}(b+m)+\frac{1}{2} \alpha X_{\alpha}(b+m)-2 \xi m X_{\alpha}(b+m)=-\frac{(b+m)}{\alpha} X_{\alpha}(b+m)$

${ }^{5}$ The commutativity of $Y_{1}(0)$ with $\left(\pi^{0}, W\right)$ for $\mathrm{g}=\hat{\mathfrak{N} S}$ was known [5] 
$+m \alpha X_{\alpha}(b+m)-2 \xi m X_{\alpha}(b+m)$ by (27). Similarly for $m<0$. Therefore, $\left[e_{m}^{-}(\xi), Y_{\alpha}(b)\right]_{+}=\left[m\left(\alpha-2 \xi-\frac{1}{\alpha}\right)-\frac{b}{\alpha}\right] X_{\alpha}(b+m)$. Now,

$$
X_{\alpha}(m)\left(1 \otimes e^{\beta T} \otimes 1\right)=1 \otimes e^{(\alpha+\beta) T} \otimes 1 \neq 0 \quad \text { if } \quad m=-\alpha \beta-\frac{\alpha^{2}}{2} .
$$

Furthermore the above implies that $\left[e_{n}^{+}(\xi), Y_{\alpha}(0)\right]=0$ for all $n \in \mathbb{Z}$. The result now follows for $\mathfrak{g}=\hat{\mathfrak{n s}}$. The proof for $\mathfrak{g}=\hat{\mathfrak{x}}$ is similar. Q.E.D.

We now use the operators $Y_{\alpha}(0)$ to construct n-invariant vectors, that is, vectors that are annihilated by $\mathrm{n}$.

Set, for $\alpha \in \mathbb{C} \backslash\{0\}$,

$$
E^{ \pm}(\alpha, \zeta)=\exp \left(-\sum_{n \in \mathbb{N}} \frac{\alpha T( \pm n)}{ \pm n} \zeta^{ \pm n}\right)
$$

and let

$$
E^{-}(\alpha, \zeta)=\sum_{n \geqq 0} f_{-n} \zeta^{-n}
$$

where $f_{-n} \in \mathbb{C}[\alpha T(-1), \alpha T(-2), \ldots]$ is a polynomial of $D$-degree $-n$ [see (3)].

Proposition 4.5. (1) The polynomials $f_{-1}, f_{-2}, \ldots$ are algebraically independent;

(2) $\mathbb{C}[\alpha T(-1), \alpha T(-2), \ldots]=\mathbb{C}\left[f_{-1}, f_{-2}, \ldots\right]$.

Proof. For a partition $v=\left(n_{1}, \ldots, n_{l}\right)$ of $n$ we write $\alpha_{v}=\alpha T\left(-n_{1}\right) \ldots \alpha T\left(-n_{l}\right)$ and proceed by induction on the weight $|v|=\sum_{j=1}^{l} n_{j}$ of $v$. By the induction assumption we see that $\alpha_{v} \in \mathbb{C}\left[f_{-1}, f_{-2}, \ldots\right]$ for all $v \neq(n)$ such that $|v|=n$. Now, $f_{-n}$ $=\frac{\alpha T(-n)}{n}+\sum_{v} c_{v} \alpha_{v}$, where $c_{v} \in \mathbb{C}$ and the sum is over all partitions $v$ such that $|v|=n$ but $v \neq(n)$. Therefore $\alpha_{v} \in \mathbb{C}\left[f_{-1}, f_{-2}, \ldots\right]$ for all $v$. This implies that the subspace $U_{-n}$ of degree $-n$ in $\mathbb{C}[\alpha T(-1), \alpha T(-2), \ldots]$ is generated by the $p(n)$ monomials in the $f_{-k}$. Since $\operatorname{dim}\left(U_{-n}\right)=p(n)$, these monomials are linearly independent. This proves (1) and (2). Q.E.D.

Let $\alpha, w \in \mathbb{C}, R \in \mathbb{N}$, and let $\zeta_{1}, \ldots, \zeta_{R}$ be distinct formal variables.

\section{Lemma 4.6.}

$$
\begin{aligned}
& X\left(\alpha_{1}, \zeta_{1}\right) \ldots X\left(\alpha, \zeta_{R}\right) 1 \otimes e^{(\omega-R \alpha) T} \\
& \quad=\prod_{1 \leqq i<j \leqq R}\left(\zeta_{j}-\zeta_{i}\right)^{2 p} \prod_{i=1}^{R} \zeta_{i}^{p-q} \prod_{i=1}^{R} \exp \left(\sum_{n \in \mathbb{N}} \frac{\alpha T(-n)}{n} \zeta_{i}^{-n}\right) 1 \otimes e^{\omega T},
\end{aligned}
$$

where $\alpha^{2}=2 p \in \mathbb{Z}_{+}, \alpha w=q$.

Proof. $E^{+}\left(\alpha, \zeta_{1}\right) E^{-}\left(\alpha, \zeta_{2}\right)=\left(1-\zeta_{1} / \zeta_{2}\right)^{2 p} E^{-}\left(\alpha, \zeta_{2}\right) E^{+}\left(\alpha, \zeta_{1}\right)$ by the BakerCampbell-Hausdorff formula. Also,

$$
\left(\zeta^{-\alpha T} e^{\alpha T}\right) e^{X}=\left(e^{\alpha T} \zeta^{-\alpha T-\alpha^{2}}\right) e^{X} \text { for all } \alpha \in \mathbb{C}, X \in \mathfrak{t} \text {, by (24). }
$$

The result now follows by recurrence. Q.E.D. 
Let $\Delta=\prod_{1 \leqq i<j \leqq R}\left(\zeta_{j}-\zeta_{i}\right)$, the Vandermonde determinant. Let $p=\frac{\alpha^{2}}{2}, q=\alpha w$, $\Delta^{2 p}=\sum_{\mu} a_{\mu} \zeta^{\mu}$, where $\mu=\left(m_{1}, \ldots, m_{R}\right), \zeta^{\mu}=\zeta_{1}^{m_{1}} \ldots \zeta_{R}^{m_{R}}$.

Proposition 4.7. $X_{\alpha}\left(n_{1}\right) \ldots X_{\alpha}\left(n_{R}\right) 1 \otimes e^{(\omega-R \alpha) T} \neq 0$ if and only if there exist $s_{1}, \ldots, s_{R} \in \mathbb{Z}_{+}$such that $\sum_{v} a_{v} \neq 0$, where $v=\left(q-p+n_{1}+s_{\pi_{1}}, \ldots, q-p+n_{R}+s_{\pi_{R}}\right)$ and $\pi$ ranges over the set $S_{R}$ of all permutations in $R$ elements.

Proof. $X_{\alpha}\left(n_{1}\right) \ldots X_{\alpha}\left(n_{R}\right) 1 \otimes e^{\omega-R \alpha}$ is the coefficient of $\zeta_{1}^{n_{1}} \ldots \zeta_{R}^{n_{R}}$ in $X\left(\alpha, \zeta_{1}\right) \ldots X\left(\alpha, \zeta_{R}\right) 1 \otimes e^{(\omega-R \alpha) T}$. By Lemma 4.6, the coefficient of $f_{-s_{1}} \ldots f_{-s_{R}} \otimes e^{\omega T}$ in $X_{\alpha}\left(n_{1}\right) \ldots X_{\alpha}\left(n_{R}\right) 1 \otimes e^{(\omega-R \alpha) T}$ is a non-zero multiple of $\sum_{v} a_{v}$ with $v$ as in the statement of the Proposition. The result now follows from Proposition 4.5 (1). Q.E.D.

Corollary 4.8. Let $R \in \mathbb{N}, \alpha^{2}=2 p$, where $P=p-\frac{1}{2}$ is a prime number, and $\alpha w=q \in \mathbb{Z}$ if $\mathfrak{g}=\hat{\mathfrak{t}} \hat{\mathfrak{s}}, \quad \alpha w=q \in \mathbb{Z}+\frac{1}{2} \quad$ if $\mathfrak{g}=\hat{\mathfrak{r}}$. Then $Y_{\alpha}(0)^{R}\left(1 \otimes e^{(\omega-R \alpha) T} \otimes 1\right) \neq 0$, and $Y_{\alpha}(0)^{R}\left(1 \otimes e^{(\omega-R \alpha) T} \otimes a(0)\right) \neq 0 \quad$ if $\mathfrak{g}=\hat{\mathfrak{r}}$, provided that $p>R+\frac{1}{2}$, and that $R p-q-\frac{R}{2} \geqq 0$ if $\mathfrak{g}=\widehat{\pi \mathfrak{s}}, R p-q-\frac{R}{2}-\frac{1}{2} \geqq 0$ if $\mathfrak{g}=\hat{\mathfrak{x}}$.

Proof. Let $\mathfrak{g}=\hat{\mathrm{n}} \hat{\mathfrak{s}}$. The coefficient of $a\left(-\frac{1}{2}\right) a\left(-\frac{3}{2}\right) \ldots a\left(-R+\frac{1}{2}\right)$ in $Y_{\alpha}(0)^{R} 1 \otimes e^{(\omega-R \alpha) T} \otimes 1$ is $X_{\alpha}\left(\frac{1}{2}\right) X_{\alpha}\left(\frac{3}{2}\right) \ldots X_{\alpha}\left(R-\frac{1}{2}\right)\left(1 \otimes e^{(\omega-R \alpha) T}\right)$. Set $S=R p-q$ $-\frac{R}{2} \in \mathbb{Z}_{+}$. The coefficient of $f_{-S}^{R} \otimes e^{\omega T}$ in $X_{\alpha}\left(\frac{1}{2}\right) \ldots X_{\alpha}\left(R-\frac{1}{2}\right)\left(1 \otimes e^{(\omega-R \alpha) T}\right)$ is $a_{v}$, where the coefficient $a_{v}$ is as in Proposition 4.7 and $v=(P(R-1), P(R-1)$ $+1, \ldots, P(R-1)+R-1)$. From the elementary properties of determinants one sees that the coefficient of $\zeta_{1}^{R-1} \ldots \zeta_{R}^{R-1}$ in $\Delta^{2}$ is $\pm R$ ! Now,

$$
\Delta^{2 P+1}=\Delta^{2 P} \sum_{\pi \in S_{R}} \operatorname{sgn}(\pi) \zeta_{\pi_{1}}^{0} \zeta_{\pi_{2}}^{1} \ldots \zeta_{\pi_{R}}^{R-1}, \Delta^{2 P} \equiv \Delta^{2}\left(\zeta_{1}^{P}, \ldots, \zeta_{R}^{P}\right)(\bmod P)
$$

and the coefficient of $\zeta_{1}^{P(R-1)} \ldots \zeta_{R}^{P(R-1)}$ in $\Delta^{2 P}$ is $\pm R !(\bmod P)$. Hence, $a_{v} \neq 0$, for $v=(P(R-1), P(R-1)+1, \ldots, P(R-1)+R-1)$ if $P>R$. For $\mathrm{g}=\hat{\mathfrak{x}}$, the argument is the same except for the following changes: Instead of $a\left(-\frac{1}{2}\right) \ldots a\left(-R+\frac{1}{2}\right)$, $X_{\alpha}\left(\frac{1}{2}\right) \ldots X_{\alpha}\left(R-\frac{1}{2}\right)\left(1 \otimes e^{\omega-R \alpha}\right) \quad$ and $\quad S=R p-q-\frac{R}{2}, \quad$ take $a(-1) \ldots a(-R)$ [respectively $\quad a(-1) \ldots a(-R) a(0)], \quad X_{\alpha}(1) \ldots X_{\alpha}(R) 1 \otimes e^{\omega-R \alpha} \quad[$ respectively $\left.X_{\alpha}(1) \ldots X_{\alpha}(R) 1 \otimes e^{\omega-R \alpha} \otimes a(0)\right] \quad$ and $\quad S=R p-q-\frac{R}{2}-\frac{1}{2} \in \mathbb{Z}_{+}, \quad$ respectively. Q.E.D.

Next, we will prove the determinant formulas $3(8)$ and $3(9)$. The proof will consist of two parts: the calculation of the $h$-degree of $\operatorname{det}(,)_{h, c, n, \gamma}$ and the divisibility of $\operatorname{det}(,)_{h, c, h, \gamma}$ by $\psi_{r, s}(h, c)^{\hat{p}(n-2 r s)}$. We note that formula $3(9)$ is obvious if $n=0$, so we assume that $n>0$. From now on $V(\lambda)=\mathbb{C}(\lambda)$ or $V(\lambda)=R(\lambda)$.

For $n \in \frac{1}{2} \mathbb{N}$, let $\mathscr{P}_{n}$ be the set of all $(d+f)$-tuples $\mathbf{I}=\left(i_{1}, \ldots, i_{d} ; j_{1}, \ldots, j_{f}\right)$ in $\mathbb{N}^{d} \times Z^{f}$ such that $i_{1} \geqq \ldots \geqq i_{d}, j_{1}>\ldots>j_{f}, j_{k}>0, k=1, \ldots, f$, and $|\mathbf{I}|=i_{1}+\ldots+i_{d}$ $+j_{1}+\ldots+j_{f}=n$. Let $\mathscr{P}_{n}^{e}$ (respectively $\mathscr{P}_{n}^{0}$ ) be the subset of $\mathscr{P}_{n}$ consisting of those 
$\mathbf{I}=\left(i_{1}, \ldots, i_{d} ; j_{1}, \ldots, j_{f}\right)$ with $f$ even (respectively $f$ odd). We say that $\mathbf{I}$ as above is equal to $\mathbf{I}^{\prime}=\left(i_{1}^{\prime}, \ldots, i_{d^{\prime}}^{\prime} ; j_{1}^{\prime}, \ldots, j_{f^{\prime}}^{\prime}\right) \in \mathscr{P}_{n}$ if $d=d^{\prime}, f=f^{\prime}$, and $i_{1}=i_{1}^{\prime}, \ldots, i_{d}=i_{d}^{\prime}$, $j_{1}=j_{1}^{\prime}, \ldots, j_{f}=j_{f^{\prime}}^{\prime}$. Let $\lambda \in \mathfrak{h}^{*}$ and set $h=\lambda\left(e_{0}^{+}\right), \quad c=\lambda\left(e_{0}^{\prime}\right)$. If $\mathbf{I} \in \mathscr{P}_{n}$, set $e_{\mathbf{1}}=e_{-i_{1}}^{+} \ldots e_{-i_{d}}^{+} e_{-j_{1}}^{-} \ldots e_{-j_{f}}^{-}$. We now consider the basis of $M(\mathbb{C}(\lambda))^{\lambda-n \delta}$ formed by the elements $e_{1} v_{\lambda}$ and the basis of $M(R(\lambda))_{0}^{\lambda-n \delta}$ [respectively $M(R(\lambda))_{1}^{\lambda-n \delta}$ ] consisting of the elements $e_{\mathbf{I}} v_{\lambda}, e_{\mathbf{K}} e_{0}^{-} v_{\lambda}$, where $\mathbf{I} \in \mathscr{P}_{n}^{e}, \mathbf{K} \in \mathscr{P}_{n}^{0}$ (respectively $\mathbf{I} \in \mathscr{P}_{n}^{0}$, $\mathbf{K} \in \mathscr{P}_{n}^{e}$ ). [Recall from the proof of Proposition 3.6 that the number of such $e_{\mathbf{1}} v_{\lambda}$ is equal to the number of such $e_{\mathbf{K}} e_{0}^{-} v_{\lambda}$ and is equal $\frac{1}{2} \hat{p}(n)$.

If $F \in \mathbb{C}[h, c]$ we let $h-\operatorname{deg}(F)$ denote the $h$-degree of $F$, that is, the degree of $F$ regarded as a polynomial of $h$.

The following lemma is the analog for $\mathfrak{g}$ of [26, Lemma 4]:

Lemma 4.9. Let $\mathbf{I}=\left(i_{1}, \ldots, i_{d} ; j_{1}, \ldots, j_{f}\right)$ and $\mathbf{K}=\left(k_{1}, \ldots, k_{g} ; l_{1}, \ldots, l_{m}\right) \in \mathscr{P}_{n}$. Then

(1) $h$-deg $\left(e_{\mathbf{1}} v_{\lambda}, e_{\mathbf{K}} v_{\lambda}\right)_{h, c, n, \gamma} \leqq \min \{d+f, g+m\}$,

$h$-deg $\left(e_{\mathbf{I}} e_{0}^{-} v_{\lambda}, e_{\mathbf{K}} e_{0}^{-} v_{\lambda}\right)_{h, c, n, \gamma} \leqq \min \{d+f, g+m\}+1$,

$h$-deg $\left(e_{\mathbf{r}} e_{0}^{-} v_{\lambda}, e_{\mathbf{K}} v_{\lambda}\right)_{h, c, n, \gamma} \leqq \min \{d+f, g+m\}$.

(2) If $d+f=g+m$ and $\mathbf{I} \neq \mathbf{K}$, then $h-\operatorname{deg}\left(e_{\mathbf{I}} v_{\lambda}, e_{\mathbf{K}} v_{\lambda}\right)_{h, c, n, \gamma}<d+f$ and $h$-deg $\left(e_{\mathbf{r}} e_{0}^{-} v_{\lambda}, e_{\mathbf{K}} e_{0}^{-} v_{\lambda}\right)_{h, c, n, \gamma}<d+f+1$.

(3) $h$-deg $\left(e_{\mathbf{r}} v_{\lambda}, e_{\mathbf{r}} v_{\lambda}\right)_{h, c, n, \gamma}=d+f$ and

$h$-deg $\left(e_{\mathbf{1}} e_{0}^{-} v_{\lambda}, e_{\mathbf{r}} e_{0}^{-} v_{\lambda}\right)_{h, c, n, \gamma}=d+f+1$.

Proof. Let $v \in\left\{v_{\lambda}, e_{0}^{-} v_{\lambda}\right\}$. Let $d>0$. Using the bracket relations in $\mathrm{g}$ we obtain:

$$
e_{i_{1}}^{+} e_{\mathbf{K}} v=\sum \dot{e_{\mathbf{K}^{\prime}}}\left(a_{\mathbf{K}^{\prime}} x_{M\left(i_{1}, \mathbf{K}^{\prime}\right)}+b_{\mathbf{K}^{\prime}} c\right) e_{\mathbf{K}^{\prime \prime}} v,
$$

where the sum is over all the $\mathbf{K}^{\prime}=\left(k_{1}^{\prime}, \ldots, \hat{k}_{p_{1}}^{\prime}, \ldots, \hat{k}_{p_{r}}^{\prime}, \ldots, k_{q^{\prime}}^{\prime} ; l_{1}^{\prime}, \ldots, \hat{l}_{q_{1}}^{\prime}, \ldots . \hat{l}_{q_{t}}^{\prime}\right)$ such that $\quad k_{p_{1}}^{\prime}+\ldots+k_{p_{r}}^{\prime}+l_{q_{1}}^{\prime}+\ldots+l_{q_{t}-1}^{\prime}<i_{1}, \quad k_{p_{1}}^{\prime}+\ldots+k_{p_{r}}^{\prime}+l_{q_{1}}^{\prime}+\ldots+l_{q_{t}}^{\prime} \geqq i_{1}$, $\mathbf{K}^{\prime \prime}=\left(l_{q_{t+1}}^{\prime}, \ldots, l_{m^{\prime}}^{\prime}\right)$, and $x_{M\left(i_{1}, \mathbf{K}^{\prime}\right)}=e_{M}^{+}$if $t$ is even, $x_{M\left(i_{1}, \mathbf{K}^{\prime}\right)}=e_{M}^{-}$if $t$ is odd, where $M=i_{1}-k_{p_{1}}^{\prime}-\ldots-k_{p_{r}}^{\prime}-l_{q_{1}}^{\prime}-\ldots-l_{q_{t}}^{\prime}, a_{\mathbf{K}^{\prime}}, b_{\mathbf{K}^{\prime}} \in \mathbb{C}$. Since $(,)_{\lambda}$ is symmetric we may assume that $k=\operatorname{deg} e_{\mathbf{I}} v \leqq \operatorname{deg} e_{\mathbf{K}} w$. The lemma now follows immediately from 3(6) and (31) by induction on $k$. Similarly if $d=0, f>0$. Q.E.D.

Proposition 4.10. For $i \in \frac{1}{2} \mathbb{N}$, set $\hat{d}(i)=\#\left\{(r, s) \mid 2 r s=i, r, s \in \frac{1}{2} \mathbb{N}, r-s \in \mathbb{Z}\right\}$ if $\mathfrak{g}=\hat{\mathbb{r}} \hat{\mathfrak{S}}$, and $\hat{d}(i)=\#\left\{(r, s) \mid 2 r s=i, r, s \in \frac{1}{2} \mathbb{N}, r-s \in \frac{1}{2}+\mathbb{Z}\right\}$ if $\mathfrak{g}=\hat{\mathfrak{r}}$. Then

$$
\begin{aligned}
& \text { (1) } h \text {-deg det }(,)_{h, c, n}=\sum_{\substack{i \in \frac{1}{2} \mathbb{N} \\
i \leq n}} \hat{p}(n-i) \hat{d}(i) \quad \text { if } \quad \mathfrak{g}=\hat{\pi} \mathfrak{s} ; \\
& \text { (2) } h \text {-deg det }(,)_{h, c, n, \gamma}=\frac{1}{2} \hat{p}(n)+\sum_{i=1}^{n} \hat{p}(n-i) \hat{d}(i) \text { if } \mathfrak{g}=\hat{\mathfrak{r}} .
\end{aligned}
$$

Proof. Let $N=\frac{1}{2} \hat{p}(n)$, and let $y_{1}, \ldots, y_{N}$ be the elements $\left\{e_{\mathbf{1}} v_{\lambda}\right\}, \mathbf{I} \in \mathscr{P}_{n}^{e}$ (respectively $\left.\mathbf{I} \in \mathscr{P}_{n}^{0}\right)$ in some order and $y_{N+1}, \ldots, y_{2 N}$ be the elements $\left\{e_{\mathbf{K}} e_{0}^{-} v_{\lambda}\right\}, \mathbf{K} \in \mathscr{P}_{n}^{0}$ (respectively $\left.\mathbf{K} \in \mathscr{P}_{n}^{e}\right)$ in some order, so that $y_{1}, \ldots, y_{2 N}$ is a basis of $M(R(\lambda))^{\lambda-n \delta}$ [respectively $\left.M(R(\lambda))_{1}^{\lambda-n \delta}\right]$. Now $\operatorname{det}(,)_{h, c, n, \gamma}=\sum_{\sigma \in S_{2 N}} \operatorname{sgn}(\sigma) \prod_{i=1}^{2 N}\left(y_{i}, y_{\sigma(i)}\right)_{h, c, n, \gamma}$. Then, by Lemma 4.9 the term corresponding to the identity permutation is the only term of $h$-degree $\frac{1}{2} \hat{p}(n)+\sum_{k=1}^{n} k \hat{p}(n, k)$, hence, by the same lemma, $h$-deg $\operatorname{det}(,)_{h, c, n, \gamma}=\frac{1}{2} \hat{p}(n)+\sum_{k=1}^{n} k \hat{p}(n, k) . \quad$ Here, $\quad \hat{p}(n, k)=\#\left\{\mathbf{I}=\left(i_{1}, \ldots, i_{d}\right.\right.$; 

$\left.\left.j_{1}, \ldots, j_{f}\right) \in \mathscr{P}_{n} \mid d+f=k\right\}$, for $\mathfrak{g}=\hat{\mathfrak{x}}$. Similarly, $h-\operatorname{deg} \operatorname{det}(,)_{h, c, n}=\sum_{k=1}^{n} k \hat{p}(n, k)$, if
$\mathfrak{g}=\hat{\mathfrak{T S}}$. Now,

$$
\frac{\prod_{n \in \mathbb{N}}\left(1+x q^{n}\right)}{\prod_{n \in \mathbb{N}}\left(1-x q^{n}\right)}=1+\sum_{n \in \mathbb{N}} \sum_{k \in \mathbb{N}} \hat{p}(n, k) x^{k} q^{n} \quad \text { in } \quad \mathbb{C} \llbracket x, q \rrbracket
$$

Taking $\frac{d}{d x}$ at $x=1$ in (32), we get

$$
\left(\sum_{n \in \mathbb{N}} \frac{q^{n}}{1-q^{n}}+\sum_{n \in \mathbb{N}} \frac{q^{n}}{1+q^{n}}\right)\left(\frac{\prod_{n \in \mathbb{N}}\left(1+q^{n}\right)}{\prod_{n \in \mathbb{N}}\left(1-q^{n}\right)}\right)=\sum_{n \in \mathbb{N}}\left(\sum_{k=1}^{n} k \hat{p}(n, k)\right) q^{n} .
$$

Let, for $i \in \mathbb{N}, d(i)=\#$ of divisors of $i$, and set $\tilde{d}(i)=d(i)+\sum_{r \in \mathbb{N}, r s=i}(-1)^{s+1}$. If $i \in 2 \mathbb{N}-1$, then $\tilde{d}(i)=2 d(i)$. Noting that if $i=r s$ then $r, s \in 2 \mathbb{N}-1$ and $i=2 \frac{r}{2} s=2 r \frac{s}{2}$, we see that $\tilde{d}(i)=\hat{d}(i)$. If $i \in 2 \mathbb{N}$, then $\tilde{d}(i)=2(\#\{r \in 2 \mathbb{N} \mid r s=i, \quad s \in 2 \mathbb{N}-1\})$ $=2(\#\{r \in \mathbb{N} \mid r s=i, s \in 2 \mathbb{N}-1\}=\hat{d}(i)$. From (33) we obtain

$$
\sum_{n \in \mathbb{N}}\left(\sum_{i \in \mathbb{N}} \hat{p}(n-i) \hat{d}(i)\right) q^{n}=\sum_{n \in \mathbb{N}}\left(\sum_{k=1}^{n} \hat{p}(n, k)\right) q^{n}
$$

This proves (2). Similarly, if $\mathfrak{g}=\hat{\mathfrak{t s}}$,

$$
\frac{\prod_{n \in \mathbb{N}-\frac{1}{2}}\left(1+x q^{n}\right)}{\prod_{n \in \mathbb{N}}\left(1-x q^{n}\right)}=\sum_{n \in \frac{1}{2} \mathbb{N}} \sum_{k \in \mathbb{N}} \hat{p}(n, k) x^{k} q^{n}, \quad \text { where } \hat{p}(n, k)
$$

is as before, Taking $\frac{d}{d x}$ at $x=1$ in (35) we obtain

$$
\left(\sum_{n \in \mathbb{N}} \frac{q^{n}}{1-q^{n}}+\sum_{n \in \mathbb{N}-\frac{1}{2}} \frac{q^{n}}{1+q^{n}}\right) \frac{\prod_{n \in \mathbb{N}-\frac{1}{2}}\left(1+q^{n}\right)}{\prod_{n \in \mathbb{N}}\left(1-q^{n}\right)}=\sum_{n \in \frac{1}{2} \mathbb{N}}\left(\sum_{k \in \mathbb{N}} k \hat{p}(n, k)\right) q^{n}
$$

Let, for $i \in \frac{1}{2} \mathbb{N}, \tilde{d}(i)=\#\left\{(r, s) \in \mathbb{N}^{2} \mid r s=i\right\}+\sum_{r \in \mathbb{N}-\frac{1}{2}, r_{\tilde{d}}=i, s \in \mathbb{N}}(-1)^{s+1}$. If $i \in \mathbb{N}$, then

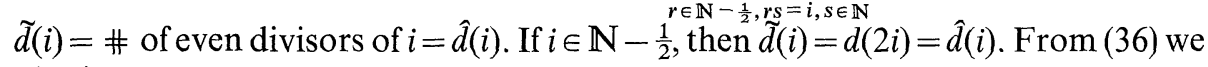
obtain

$$
\sum_{n \in \frac{1}{2} \mathbb{N}}\left(\sum_{i \in \frac{1}{2} \mathbb{N}} \hat{p}(n-i) \hat{d}(i)\right) q^{n}=\sum_{n \in \frac{1}{2} \mathbb{N}}\left(\sum_{k \in \mathbb{N}} k \hat{p}(n, k)\right) q^{n}
$$

Hence $\sum_{i \in \frac{1}{2} \mathbb{N}} \hat{p}(n-i) \hat{d}(i)=\sum_{k \in \mathbb{N}} k \hat{p}(n, k)$. This proves (1). Q.E.D.

Lemma 4.11. Let $r, s \in \frac{1}{2} \mathbb{N}$ be such that $s-r \in \mathbb{Z}_{+}$if $\mathfrak{g}=\widehat{\pi \mathfrak{s}}$ and $s-r \in \frac{1}{2}+\mathbb{Z}_{+}$if $\mathfrak{g}=\hat{\mathfrak{r}}$. Let $p \in \frac{1}{2}+\mathbb{N}$ be such that $p-\frac{1}{2}$ is prime and $p>2 r+\frac{1}{2}$. Let $c=1-\frac{(2 p-1)^{2}}{p}$ 
$\gamma \in \mathbb{Z}_{2}$, and $h=\frac{(2 p-1)^{2}-4(2 r p-s)^{2}}{16 p}$ if $\mathfrak{g}=\mathfrak{n s}, h=\frac{2(p-1)^{2}-4(2 r p-s)^{2}}{16 p}-\frac{1}{16}$ if $\mathfrak{g}=\hat{\mathfrak{r}}$. Then $\operatorname{det}(,)_{h, c, 2 r s, \gamma}=0$.

Proof. Let $\xi=\frac{2 p-1}{2 \sqrt{2 p}}, \omega=\frac{2 r p-s}{\sqrt{2 p}}, \alpha=\sqrt{2 p}$ and $R=2 r$. Then $\xi=\frac{\alpha^{2}-1}{2 \alpha}$, and so $Y_{\alpha}(0)^{R}$ intertwines the action of $\mathfrak{g}$ on $\mathscr{K}^{\xi, \omega-R \alpha}$ and $\mathscr{K}^{\xi, \omega}$ by Theorem 4.4. Therefore, by Theorems 4.2 and 4.4, and by Proposition 4.3, $u_{\omega, \alpha, R}$ $=Y_{\alpha}(0)^{R}\left(1 \otimes e^{(\omega-R \alpha) T} \otimes 1\right)$ and $e_{0}^{-} u_{\omega, \alpha, R}$ are $n$-invariant vectors of $\left(\mathscr{K}^{\xi, \omega}\right)^{\mu}$, where $\mu\left(e_{0}^{\prime}\right)=1-8 \xi^{2}$ and $\mu\left(e_{0}^{+}\right)=\frac{\xi^{2}-\omega^{2}}{2}-2 r s$ if $\mathfrak{g}=\hat{\mathfrak{r s}}, \mu\left(e_{0}^{+}\right)=\frac{\xi^{2}-\omega^{2}}{2}-\frac{1}{16}-2 r s$ if $\mathfrak{g}=\hat{\mathfrak{r}}$. Let $q=2 r p-s$. Then $q \in \mathbb{Z}$ and $R p-q-\frac{R}{2} \geqq 0$ if $\mathfrak{g}=\hat{\mathfrak{r} s}$, and $q \in \mathbb{Z}+\frac{1}{2}$ and $R p-q-\frac{R}{2}-\frac{1}{2} \geqq 0$ if $\mathfrak{g}=\hat{\mathfrak{r}}$. Also note that $\alpha^{2}=2 \mathrm{p}$ and $\alpha w=q$. By Corollary 4.8, $u_{\omega, \alpha, R}=Y_{\alpha}(0)^{R}\left(1 \otimes e^{(\omega-R \alpha) T} \otimes 1\right)$ and $e_{0}^{-} u_{\omega, \alpha, R}$ are both nonzero. Let $\mathscr{U}$ denote the $\mathrm{g}$-submodule of $\mathscr{K}^{\xi, \omega}$ generated by $1 \otimes e^{\omega T} \otimes 1$. By the above and Proposition 3.7 there is a unique surjective homomorphism $\phi: M(V(\lambda)) \rightarrow \mathscr{U}$ such that $\phi\left(v_{\lambda}\right)$ $=1 \otimes e^{\omega T} \otimes 1$, since $c=1-8 \xi^{2}$, and $h=\frac{\xi^{2}-\omega^{2}}{2}$ if $\mathfrak{g}=\hat{\mathfrak{r} \mathfrak{s}}, h=\frac{\xi^{2}-\omega^{2}}{2}-\frac{1}{16}$ if $\mathfrak{g}=\hat{\mathfrak{r}}$. Let $\psi=i \circ \phi: M(V(\lambda)) \rightarrow \mathscr{K}^{\xi, \omega}$, where $i: \mathscr{U} \rightarrow \mathscr{K}^{\xi, \omega}$ is the inclusion map. If $u_{\omega, \alpha, R} \in \operatorname{Im} \psi=\mathscr{U}$ and if $e_{0}^{-} u_{\omega, \alpha, R} \in \operatorname{Im} \psi$, then the assertion follows from Proposition 3.14. If $u_{\omega, \alpha, R} \notin \operatorname{Im} \psi$, then $\operatorname{dim}(\operatorname{Im} \psi)^{\frac{\lambda}{0}-2 r s \delta}<\hat{p}(2 r s)=\operatorname{dim} M(V(\lambda))^{\frac{\lambda}{0}-2 r s \delta}$. Therefore, $(\operatorname{Ker} \psi)^{\lambda-2 r s \delta} \neq(0)$, and so, $\operatorname{det}(,)_{\lambda, 2 r s, \overline{0}}=0$, by Proposition 3.14. Similarly, if $e_{0}^{-} u_{\omega, \alpha, R} \notin \operatorname{Im} \psi$. Q.E.D.

Let $\psi_{r, s}$ be as in Sect. 3 if $r, s \in \frac{1}{2} \mathbb{N}$, and let $\psi_{0,0}(h, c)=h+\frac{c}{16}$ if $\mathfrak{g}=\hat{\mathfrak{r}}$. Let $\mathscr{V}_{r, s}$ denote the variety in $\mathbb{C}^{2}$ consisting of all points $(h, c)$ such that $\psi_{r, s}(h, c)=0$, $(r, s) \in \frac{1}{2} \mathbb{N} \times \frac{1}{2} \mathbb{N} \cup\{(0,0)\}$.

Lemma 4.12. Let $r, s \in \frac{1}{2} \mathbb{N}, r \leqq s$. Then
(1) $\mathscr{V}_{r, s}=\left\{\left(\frac{(2 p-1)^{2}-4(2 p r-s)^{2}}{16 p}, 1-\frac{(2 p-1)^{2}}{p}\right) \mid p \in \mathbb{C} \backslash\{0\}\right\} \quad$ if $\mathfrak{g}=\hat{\mathfrak{r s}}$;
(2) $\mathscr{V}_{r, s}=\left\{\left(\frac{(2 p-1)^{2}-4(2 p r-s)^{2}}{16 p}-\frac{1}{16}, 1-\frac{(2 p-1)^{2}}{p}\right) \mid p \in \mathbb{C} \backslash\{0\}\right\}$ if $\mathfrak{g}=\mathfrak{r}$

Proof. Let $p^{ \pm}=\frac{5-c \pm \sqrt{c^{2}-10 c+9}}{8}$. Then $\left(2 p^{ \pm}-1\right)^{2}=p^{ \pm}(1-c)$, i.e. $c=1$ $-\frac{\left(2 p^{ \pm}-1\right)^{2}}{p^{ \pm}}$. Now, $h=h_{r, s}^{ \pm}(c) \Leftrightarrow h=\frac{1-c}{16}-r^{2} p^{ \pm}+r s-\frac{s^{2}}{4 p^{ \pm}} \quad$ if $\quad \mathfrak{g}=\hat{\mathfrak{s}}, \quad$ and $h=\frac{1-c}{16}-r^{2} p^{ \pm}+r s-\frac{s^{2}}{4 p^{ \pm}}-\frac{1}{16}$ if $\mathfrak{g}=\hat{\mathfrak{x}}$. Hence $h=h_{r, s}^{ \pm}(c) \Leftrightarrow h=\frac{1}{16 p^{ \pm}}\left[(1-c) p^{ \pm}\right.$ $\left.-4\left(2 r p^{ \pm}-s\right)^{2}\right]$ if $\mathfrak{g}=\widehat{\mathfrak{n} \mathfrak{s}}, h=\frac{1}{16 p^{ \pm}}\left[(1-c) p^{ \pm}-4\left(2 r p^{ \pm}-s\right)^{2}\right]-\frac{1}{16}$ if $\mathfrak{g}=\hat{\mathfrak{r}}$. Let $\psi_{r, s}(h, c)=0 . \quad$ Then $\quad h=h_{r, s}^{ \pm}(c) . \quad$ Therefore, $\quad c=1-\frac{(2 p-1)^{2}}{2} \quad$ and 
$h=\frac{(2 p-1)^{2}-4(2 r p-s)^{2}}{16 p}$ if $\mathfrak{g}=\hat{\kappa} \hat{\mathfrak{s}}, h=\frac{(2 p-1)^{2}-4(2 r p-s)^{2}}{16 p}-\frac{1}{16}$ if $\mathfrak{g}=\hat{\mathfrak{r}}$, where $p=p^{ \pm}$. Conversely, let $c=1-\frac{(2 p-1)^{2}}{2}$ and $h=\frac{(2 p-1)^{2}-4(2 r p-s)^{2}}{16 p}$ if $\mathfrak{g}=\hat{\mathfrak{r}}$, $h=\frac{(2 p-1)^{2}-4(2 r p-s)^{2}}{16 p}-\frac{1}{16}$ if $\mathfrak{g}=\hat{\mathrm{x}}$. Then $p=p^{ \pm}$and $h=h_{r, s}^{ \pm}$, i.e. $\psi_{r, s}(h, c)=0$. Q.E.D.

Proposition 4.13. Let $h, c \in \mathbb{C}, \gamma \in \mathbb{Z}_{2}$. Let $r, s \in \frac{1}{2} \mathbb{N}, r \leqq s$, and $r-s \in \mathbb{Z}$ if $\mathfrak{g}=\hat{\mathfrak{n}} \hat{\mathfrak{s}}$, $r-s \in \frac{1}{2}+\mathbb{Z}$ if $\mathfrak{g}=\hat{\mathfrak{r}}$. Then $\operatorname{det}(,)_{h, c, 2 r s, \gamma}$ vanishes on $\mathscr{V}_{r, s}$. Furthermore, if $\mathfrak{g}=\hat{\mathfrak{r}}$, $n \in \mathbb{Z}_{+}$, then $\operatorname{det}(,)_{h, c, n, \gamma}$ vanishes on $\mathscr{V}_{0,0}$.

Proof. Assume $(r, s) \neq(0,0)$. Let $\theta: \mathbb{C}[h, c] \rightarrow \mathbb{C}\left[p, p^{-1}\right]$ be the homomorphism defined by $\theta(c)=1-\frac{(2 p-1)^{2}}{p}$ and $\theta(h)=\frac{(2 p-1)^{2}-4(2 r p-s)^{2}}{16 p}$ if $\mathfrak{g}=\hat{\mathfrak{r}} \mathfrak{s}, \theta(h)$ $=\frac{(2 p-1)^{2}-4(2 r p-s)^{2}}{16 p}-\frac{1}{16}$ if $\mathrm{g}=\hat{\mathrm{r}}$. By Lemma 4.11, $\theta\left(\operatorname{det}(,)_{h, c, 2 r s, \gamma}\right)$ vanishes at infinitely many values of $p$. Therefore, $\theta\left(\operatorname{det}(,)_{h, c, 2 r s, \gamma}\right) \equiv 0$. This implies, by Lemma 4.12 , that $\operatorname{det}(,)_{h, c, 2 r s, \gamma}$ vanishes on $\mathscr{V}_{r, s}$. Also, if $\mathfrak{g}=\hat{\mathfrak{r}}$, we saw that $M(R(\lambda)) \supset M(S(\lambda))$ if $h=-\frac{c}{16}$ (Proposition 3.5). Hence, for each $n \in \mathbb{Z}_{+}$, $\operatorname{det}(,)_{h, c, n, \gamma}$ vanishes on $\mathscr{V}_{0,0}$. Q.E.D.

Proof of the Determinant Formulas. We proceed by induction on $n$. Let $r, s$ be as in Proposition 4.13, $n \in \mathbb{Z}_{+}, 2 r s \leqq n$. Let $u=\varphi_{r, s}(h, c), v=c$. Then $\left|\frac{\partial(u, v)}{\partial(h, c)}\right|=\frac{\partial}{\partial h}$ $\cdot \psi_{r, s}(h, c)$. Hence, if $r=s, \quad \frac{\partial(u, v)}{\partial(h, c)}=1 \neq 0, \quad$ and $\quad$ if $\quad r \neq s, \quad\left|\frac{\partial(u, v)}{\partial(h, c)}\right|=0 \Leftrightarrow h$ $=\frac{h_{r, s}^{+}(c)+h_{r, s}^{-}(c)}{2}$. Thus, at $h=h_{r, s}^{ \pm}(c),\left|\frac{\partial(u, v)}{\partial(h, c)}\right|=0 \Leftrightarrow c=1$ or $c=9$. This implies that $\{u, v\}$ is a local system of coordinates near all $(h, c) \in \mathscr{V}_{r, s} \backslash\left\{\left(h_{r, s}^{ \pm}(1), 1\right),\left(h_{r, s}^{ \pm}(9)\right.\right.$, 9)\}. By letting $n$ act on the basis of $M(V(\lambda))_{\gamma}^{\lambda-m \delta}$ described in the proof of Proposition 4.10, we see that $\operatorname{det}(,)_{h, c, m, \gamma}=0$ if and only if there is a non-zero $n$ invariant vector in $M(V(\lambda))_{\gamma}^{\lambda-q \delta}, q \leqq m$. Therefore, if $(h, c) \in \mathscr{V}_{r, s}$, then there is a non-zero nt-invariant vector in $M(V(\lambda))_{\gamma}^{\lambda-q \delta}, q \leqq 2 r s$, by Proposition 4.13. Let

$$
\mathscr{V}_{r, s}^{\prime}=\mathscr{V}_{r, s} \backslash\left(\left\{\left(h_{r, s}^{ \pm}(1), 1\right),\left(h_{r, s}^{ \pm}(9), 9\right)\right\} \underset{\substack{\left(r^{\prime}, s^{\prime}\right) \neq(r, s) \\ r^{\prime} \prime^{\prime} \leqq r s}}{\bigcup} \mathscr{V}_{r^{\prime}, s^{\prime}}\right)
$$

if $(r, s) \neq(0,0), \mathscr{V}_{0,0}^{\prime}=\mathscr{V}_{0,0}$. Arguing as in [23, Lemma 3.2], we see that $\mathscr{V}_{r, s}^{\prime}$ contains all but a finite number of points of $\mathscr{V}_{r, s}$. Let $\lambda_{0} \in \mathscr{V}_{r, s}^{\prime}$. By the induction hypothesis there is then a non-zero $n$-invariant vector in $M\left(V\left(\lambda_{0}\right)\right)_{\gamma}^{\lambda_{0}-2 r s \delta}$. Let $\mathscr{U}$ be the submodule of $M\left(V\left(\lambda_{0}\right)\right)$ generated by this vector. Then $\operatorname{dim}\left(M\left(V\left(\lambda_{0}\right)\right)_{\gamma}^{\lambda_{0}-n \delta}\right.$ $\cap \mathscr{U})=\check{p}(n-2 r s)$, where $\breve{p}(n-2 r s)=\hat{p}(n-2 r s)$ if $(r, s) \neq(0,0), \check{p}(n)=\frac{1}{2} \hat{p}(n)$, by Propositions 3.1 and 3.6. Therefore,

$$
\operatorname{det}_{n}(,)_{h(u, v), c(u, v), n, \gamma}=u^{\check{p}(n-2 r s)} f(u, v),
$$


where $f$ is analytic near $\left(u_{0}, v_{0}\right)$ and $u_{0}, v_{0}$ are the $(u, v)$-coordinates of $\lambda_{0}$. Let $g(h, c)$ be an irreducible divisor of $\operatorname{det}(,)_{h, c, n, \gamma}$ in $\mathbb{C}[h, c]$. If $g(h(u, v), c(u, v))=u k(u, v)$, with $k$ analytic near $\left(u_{0}, v_{0}\right)$, then $g(h, c)=0$ for infinitely many $(h, c)$ in $\mathscr{V}_{r, s}$. Hence $\theta(g(h, c))=0$ for all $(h, c)$ and $g(h, c)=0$ for all $(h, c)$ on $\mathscr{V}_{r, s}$ as in the proof of Proposition 4.13. Thus $g(h, c)$ is a nonzero multiple of $\psi_{r, s}(h, c)$. This, (38) and Proposition 13 show that $\psi_{r, s}(h, c)^{\check{p}^{(n-2 r s)}}$ divides $\operatorname{det}(,)_{h, c, n, \gamma}$. The degree computation of Proposition 4.10 gives (8) and (9). Q.E.D.

\section{The Irreducible Subquotients of a Verma Module}

We retain the notation of Sect. 3 .

For $n \in \frac{1}{2} \mathbb{N}$, let $\phi_{n}(h, c)=\prod_{2 r s=n} \psi_{r, s}(h, c)$, where the product is over all $r, s \in \frac{1}{2} \mathbb{N}$ such that $s-r \in \mathbb{N}$ if $\mathfrak{g}=\hat{\mathfrak{n} \mathfrak{s}}, s-r \in \mathbb{N}-\frac{1}{2}$ if $\mathfrak{g}=\hat{\mathfrak{r}}$. As in [22], we set, for $\lambda \in \mathfrak{h}^{*}$, $\left(\frac{1}{2} \mathbb{N}\right)_{+}(\lambda)=\left\{n \in \frac{1}{2} \mathbb{N} \mid \phi_{n}(h, c)=0\right\}$. Let $\mu, \lambda \in \mathfrak{h}^{*}$. We write $\mu \uparrow \lambda$ if $\mu=\lambda$ or if $\mu=\lambda-\left(\sum_{i=1}^{r} m_{i}\right) \delta$ for some $m_{1}, \ldots, m_{r} \in \frac{1}{2} \mathbb{N}$ such that

$$
m_{1} \in\left(\frac{1}{2} \mathbb{N}\right)_{+}(\lambda), \quad m_{i} \in\left(\frac{1}{2} \mathbb{N}\right)_{+}\left(\lambda-\sum_{j=1}^{i-1} m_{j} \delta\right), \quad 2 \leqq i \leqq r .
$$

A subquotient of a module $M$ is a quotient $N / U$ where $U \subset N \subset M, U, N$ submodules of $M$. From now on, unless otherwise stated, $V(\lambda)=\mathbb{C}(\lambda)$ if $\mathfrak{g}=\hat{\mathrm{ns}}$ and $V(\lambda)=R(\lambda)$ if $\mathfrak{g}=\hat{\mathfrak{x}}$.

Theorem 5.1. Let $\lambda, \mu \in \mathfrak{h}^{*}$. If $L(V(\mu))$ is a subquotient of $M(V(\lambda))$, then $\mu \uparrow \lambda .^{6}$

In this section we prove Theorem 5.1. The construction of filtrations of Verma modules given below is a straight-forward generalization of the one given in [23].

Let $V=U\left(\mathfrak{n}^{-}\right), \mu \in \mathfrak{h}^{*}$. Let $T_{\mu}: V \rightarrow M(V(\mu))$ be the linear $\left(\mathbb{Z}_{2}\right.$-graded) isomorphism defined by $T_{\mu}(X)=X v_{\mu}, X \in V$. We set $\tau_{\mu}(Y) v=T_{\mu}^{-1}\left(Y T_{\mu}(v)\right)$ for $Y \in \mathfrak{g}, v \in V$. Then $\left(V, \tau_{\mu}\right)$ is a representation of $g$. On $V \times V$ we define $A_{\mu}(v, w)=\left(T_{\mu}(v), T_{\mu}(w)\right)_{\mu}$. It is automatic to see that $A_{\mu}$ defines a symmetric bilinear form on $V \times V$ such that

$$
A_{\mu}\left(\tau_{\mu}(Y) v, w\right)=A_{\mu}\left(v, \tau_{\mu}(\sigma(Y) w)\right)
$$

for all $Y \in U(\mathfrak{g}), v, w \in V$.

We now let $\lambda$ be fixed and for each $t \in \mathbb{C}$, we set

$$
\begin{aligned}
& \left(\pi_{t}, V\right)=\left(\tau_{\lambda+t \delta}, V\right), \\
& B_{t}(,)=A_{\lambda+t \delta}(,) .
\end{aligned}
$$

Now, $V=\bigoplus_{\eta \in Q^{+} \cup\{0\}} V^{\lambda-\eta}$.

Let $\mathcal{O}(\mathbb{C})$ denote the space of germs $\mathbf{f}$ of complex valued holomorphic functions $f$ at 0 . Let $\mathcal{O}(V)^{F}$ denote the space of germs $\mathbf{f}$ of holomorphic functions at 0 with values in a finite sum of spaces $V^{\lambda-\eta}$. For $X \in \mathfrak{g}, t$ near $0, \mathbf{f} \in \mathcal{O}(V)^{F}$, set

$$
(\pi(X) \mathbf{f})(t)=\pi_{t}(X)(f(t)) .
$$

${ }^{6}$ For $\mathfrak{g}=\hat{\pi} \mathfrak{s}$ this result was first stated in [14] 
For $k \in \mathbb{Z}_{+}$, set

$$
\left.\mathcal{O}(V)_{(k)}^{F}=\left\{\mathbf{f} \in \mathcal{O}(V)^{F} \mid B_{t}(f(t), w) \in t^{k} \mathcal{O} \mathbf{C}\right) \forall w \in V\right\} .
$$

Now, set

$$
V_{(k)}^{F}=\left\{f(0) \mid \mathbf{f} \in \mathcal{O}^{F}(V)_{(k)}\right\},
$$

and

$$
M(V(\lambda))_{(k)}=T_{\lambda}\left(V_{(k)}^{F}\right) .
$$

Clearly, $M(V(\lambda))=M(V(\lambda))_{(0)} \supset M(V(\lambda))_{(1)} \supset \ldots$ By (1), (3), $B_{t}\left(\pi_{t}(X) f(t), w\right)$ $=B_{t}(f(t), \pi(\sigma(X)) w) \in t^{k} \mathcal{O}(\mathbb{C})$ for all $\mathbf{f} \in \mathcal{O}(V)_{(k)}^{F}$, i.e., $\pi(X) \mathcal{O}(V)_{(k)} \subset \mathcal{O}(V)_{(k)}$, by $(4)$. This implies that $\pi_{0}(X) V_{(k)}^{F} \subset V_{(k)}^{F}$. Therefore, $X M(V(\lambda))_{(k)}=X T_{\lambda}\left(V_{(k)}^{F}\right)$ $=T_{\lambda}\left(\tau_{\lambda}(X) V_{(k)}^{F}\right) \subset T_{\lambda}\left(V_{(k)}^{F}\right)=M(V(\lambda))_{(k)}$. This shows that $\left(M(V(\lambda))_{(k)}\right)_{k \in \mathbb{Z}_{+}}$is a $\mathrm{g}$-module filtration of $M(V(\lambda))$. We now show that this filtration is $\mathbb{Z}_{2}$-graded. We note that $B_{t}\left(V_{\gamma_{1}}^{\eta_{1}}, V_{\gamma_{2}}^{\eta_{2}}\right)=0$ if $\gamma_{1} \neq \gamma_{2}$ or $\eta_{1} \neq \eta_{2}$. Let $P_{\gamma}: V \rightarrow V_{\gamma}$ be the obvious surjection. Let $v \in V_{(k)}^{F}$ and $\mathbf{f} \in \mathcal{O}^{F}(V)_{(k)}$ be such that $v=f(0)$. Now, $v=v_{\overline{0}}+v_{\overline{1}}, v_{\gamma} \in V_{\gamma}$ and $v_{\gamma}=f_{\gamma}(0)$, where $\mathbf{f}_{\gamma}$ is the germ of $P_{\gamma} \circ f$. Let $w \in V_{\gamma}$. Then $B_{t}(f(t), w) \in t^{k} \mathcal{O}(\mathbb{C})$. But $B_{t}(f(t), w)=B_{t}\left(f_{\gamma}(t), w\right)$ by Proposition 3.14(b). Applying Proposition 3.14(b) again we see that $B_{t}\left(f_{\gamma}(t), w\right) \in t^{k} \mathcal{O}(\mathbb{C})$ for all $w \in V$. Therefore, $f_{\gamma} \in \mathcal{O}^{F}(V)_{(k)}$ and so $v_{\gamma} \in V_{(k)}^{F}$ for all $\gamma \in \mathbb{Z}_{2}$. This proves that $\left(M(V(\lambda))_{(k)}\right)_{k \in \mathbb{Z}_{+}}$is $\mathbb{Z}_{2}$-graded. For any finite dimensional vector space $M$ we let $\mathscr{L}_{s}^{2}(M, \mathbb{C})$ (respectively $\operatorname{Sym}(M)$ ) denote the space of all symmetric bilinear forms on $M$ (respectively symmetric endomorphisms of $M)$. Let $\mathcal{O}(M)$ denote the space of germs of $M$-valued holomorphic functions at 0 . Let $B_{t}^{\eta}=B_{t \mid V^{\lambda-\eta} \times V^{\lambda-\eta}}$, and let $(\mid)_{\eta}$ be any symmetric bilinear nonsingular form on $V^{\lambda-\eta}$. Set $B^{\eta}(t)=B_{t}^{\eta}, t \sim 0$. Then $\mathbf{B}^{\eta} \in \mathcal{O}\left(\mathscr{L}_{s}^{2}\left(V^{\lambda-\eta}, \mathbb{C}\right)\right)$ and $\exists \mathbf{A}^{\eta} \in \mathcal{O}\left(\operatorname{Sym}\left(V^{\lambda-\eta}\right)\right)$ such that $B_{t}^{\eta}(v, w)=\left(A_{t}^{\eta} v \mid w\right)$ for all $v, w \in V^{\lambda-\eta}$. Here $\mathbf{A}^{\eta}(t)=A_{t}^{\eta}, t \sim 0$. Set

$$
\begin{gathered}
\mathcal{O}\left(V^{\lambda-\eta}\right)_{(k)}=\left\{\mathbf{f} \in \mathcal{O}\left(V^{\lambda-\eta}\right) \mid A_{t}^{\eta} f(t)=t^{k} g(t) \text { for some } \mathbf{g} \in \mathcal{O}\left(V^{\lambda-\eta}\right), \text { all } t \sim 0\right\}, \\
\left(V^{\lambda-\eta}\right)_{(k)}=\left\{f(0) \mid \mathbf{f} \in \mathcal{O}\left(V^{\lambda-\eta}\right)_{(k)}\right\} .
\end{gathered}
$$

Then $\left(V^{\lambda-\eta}\right)_{(k)}=V_{(k)}^{F} \cap V^{\lambda-\eta}$. Also,

$$
\operatorname{Ch} M(V(\lambda))_{(k)}=\sum_{\eta} \operatorname{dim}\left(V^{\lambda-\eta}\right)_{(k)} q^{\lambda-\eta}
$$

where $\eta \in Q^{+} \cup\{0\}$.

If $\mathbf{f} \in \mathcal{O}(\mathbb{C})$, let $f(t)=\sum_{i \geqq 0} a_{i} t^{i}, t \sim 0$. If $a_{i} \neq 0$ for at least one $i$, we can define the number $\operatorname{ord}_{0} f(t)$ as the smallest $i$ with this property. Applying Lemma 1.2 of [23] to $V^{\lambda-\eta}$ and $\mathbf{A}^{\eta}$, we obtain

$$
\sum_{k \geqq 1}\left(\operatorname{dim} V^{\lambda-\eta}\right)_{(k)}=\operatorname{ord}_{0} \operatorname{det} B_{t}^{\eta} .
$$

Combining (10) and (11) we obtain

$$
\sum_{k \geqq 1} \operatorname{ch} M(V(\lambda))_{(k)}=\sum_{\eta} q^{\lambda-\eta} \operatorname{ord}_{0} \operatorname{det} B_{t}^{\eta} .
$$


Now, $\operatorname{ord}_{0} \psi_{r, s}(h+t, c)$ is one or zero according to whether $\psi_{r, s}(h, c)=0$ or $\psi_{r, s}(h, c) \neq 0$. Let $a(i)=\{(r, s) \mid 2 r s=i\} .3(8)$ and 3(9) imply

$$
\begin{gathered}
\sum_{k \geqq 1} \operatorname{ch} M(\mathbb{C}(\lambda))_{(k)}=\sum_{i \in \frac{1}{2} \mathbb{N}_{+}(\lambda)} a(i) \sum_{\eta=n \delta} \hat{p}(n-i) q^{\lambda-\eta}, \\
\sum_{k \geqq 1} \operatorname{ch} M(R(\lambda))_{(k)}=\delta_{h,-\frac{c}{16}} \sum_{\eta=n \delta} \frac{1}{2} \hat{p}(n) q^{\lambda-\eta}+\sum_{i \in \frac{1}{2} \mathbb{N}_{+}(\lambda)} a(i) \sum_{\eta=n \delta} \hat{p}(n-i) q^{\lambda-\eta} .
\end{gathered}
$$

Therefore, we have

Proposition 5.2. The following are valid:

(1) $\sum_{k \geqq 1} \operatorname{ch} M(\mathbb{C}(\lambda))_{(k)}=\sum_{i \in \frac{1}{2} \mathbb{N}_{+}(\lambda)} a(i) \operatorname{ch} M(\mathbb{C}(\lambda-i \delta))$,

(1) $\sum_{k \geqq 1} \operatorname{ch} M(R(\lambda))_{(k)}=\delta_{h,-\frac{c}{16}} \operatorname{ch} M(S(\lambda))+\sum_{i \in \frac{1}{2} \mathbb{N}+(\lambda)} a(i) \operatorname{ch} M(R(\lambda-i \delta))$.

If $\mathbf{f}, \mathbf{g} \in \mathcal{O}\left(V^{\lambda-\eta}\right)$, set $\langle f, g\rangle(t)=B_{t}^{\eta}(f(t), g(t))$. Now, let $v, w \in V^{\lambda-\eta}, v=f(0)$, $w=g(0), \mathbf{f}, \mathbf{g} \in \mathcal{O}\left(V^{\lambda-\eta}\right)$. The argument in [23, Sect.1] shows that $\langle v, w\rangle$ $=\langle f, g\rangle(0)$ defines a symmetric, bilinear form on $V^{\lambda-\eta}$ which factors to a symmetric, bilinear, nondegenerate form on $V^{\lambda-\eta} /\left(V^{\lambda-\eta}\right)_{(1)}([23$, Lemma 1.3]). This implies that $M(V(\lambda))_{(1)}$ is the maximal proper submodule of $M(V(\lambda))$.

If $M$ is a weight module, $M=\bigoplus_{\mu} M^{\mu}$, we set $P(M)=\left\{\mu \in \mathfrak{h}^{*} \mid M^{\mu} \neq(0)\right\}$. Let $\mathscr{C}$ be the full subcategory of the category of all $\mathfrak{g}$-modules $M$ such that

$$
\begin{gathered}
M=\bigoplus_{\mu \in \mathfrak{h}^{*}} M^{\mu}, \operatorname{dim} M^{\mu}<\infty, \\
P(M) \subset\left\{v-\left(Q^{+} \cup\{0\}\right)\right\} \text { for some } v \in \mathfrak{h}^{*} .
\end{gathered}
$$

If $\mu_{1}, \mu_{2} \in \mathfrak{h}^{*}$ we set $\mu_{1}<\mu_{2} \Leftrightarrow \mu_{2}-\mu_{1}>0$. Arguing as in [9, Lemma 4.4] (see also [24, Lemma 5.1]) we obtain

Lemma 5.3. Let $M \in \mathscr{C}$. For each $i \in \mathbb{Z}_{+}$there is a submodule ${ }_{i} M$ of $M$ such that $(0)={ }_{0} M \subset{ }_{1} M \subset \ldots, \bigcup_{i=0}^{\infty}{ }_{i} M=M,{ }_{i} M /{ }_{i-1} M$ is a highest weight module with highest weight $v_{i} \in \mathfrak{h}^{*}$. Furthermore, $\mu_{i}<\mu_{j} \Rightarrow i>j$, and if $v_{i}=v_{i+1}$, then $\operatorname{dim}\left({ }_{i} M /{ }_{i-1} M\right)^{v_{i}}$ $>\operatorname{dim}\left({ }_{i+1} M /{ }_{i} M\right)^{v_{i}}$.

Using Lemma 5.3 we can define for each $M \in \mathscr{C}$ the multiplicity $(M: L(V(\mu)))$ as in [24, Sect. 5] by first defining $(M: L(V(\mu)))$ for a highest weight module $M$ of $\mathscr{C}$. The only distinction here is in the case $\mathfrak{g}=\hat{\mathrm{r}}$ : If $M$ is a highest weight module with highest weight $\lambda=(h, c)$ such that $\operatorname{dim} M^{\lambda}=2$ and $\lambda\left(e_{0}^{+}\right)+\frac{\lambda\left(e_{0}^{\prime}\right)}{16}=0$, then we set $(M: L(V(\lambda)))=2$. Otherwise, $(M: L(V(\lambda)))=1$, for a highest weight module of highest weight $\lambda$.

Proposition 5.4. Let $M \in \mathscr{C}$. Then

$$
\operatorname{ch} M=\sum_{\mu}(M: L(V(\mu))) \operatorname{ch} L(V(\mu)) .
$$

Proof. We will show that

$$
\operatorname{dim} M^{\xi}=\sum_{\mu}(M: L(V(\mu))) \operatorname{dim} L(V(\mu))^{\xi} \text { for all } \xi \in \mathfrak{h}^{*} .
$$


Let $M$ be a highest weight module with highest weight $v$. If $M=L(V(v))$, then $(M: L(V(\mu)))=1$ if $\mu=v$ and $(M: L(V(\mu)))=0$, otherwise. So, we may assume that $M$ is reducible. Now, $\operatorname{dim} L\left(V\left(v-\eta^{\prime}\right)\right)^{v} \neq 0$ only if $\eta^{\prime}=0$. Furthermore, if $\operatorname{dim} M^{v}=2$, then either $v\left(e_{0}^{+}\right)+\frac{1}{16} v\left(e_{0}^{\prime}\right)=0$ and $\operatorname{dim} L(R(v))^{v}=\operatorname{dim} L(S(v))^{v}=1$ (see Proposition 3.10), or $v\left(e_{0}^{+}\right)+\frac{1}{16} v\left(e_{0}^{\prime}\right) \neq 0$ and $\operatorname{dim} L(R(v))^{v}=2$.

In any case, for $\xi=v,(17)$ follows from the definition of $(M: L(V(\mu)))$. Assume that (17) was proved for all $\xi=v-\eta^{\prime}$, with $\eta^{\prime}<\eta$. There are two cases to consider:

1) $\operatorname{dim} M^{\nu}=1$. Let $\left\{{ }_{i} M_{(1)}\right\}_{i=0}^{\infty}$ be a filtration of $M_{(1)}$ as in Lemma 3.5, and let $s$ be the largest index such that $\left({ }_{s} M_{(1)} / s+1 M_{(1)}\right)^{v-\eta} \neq(0)$. Now, $\left.\operatorname{dim} M^{v-\eta}=\operatorname{dim} L V(v)\right)^{v-\eta}+\operatorname{dim} M_{(1)}^{v-\eta} \quad$ and $\quad \operatorname{dim} M_{(1)}^{v-\eta}=\operatorname{dim}\left({ }_{1} M_{(1)}^{v-\eta}\right)$ $+\operatorname{dim}\left(\left({ }_{2} M_{(1)} / 1 M_{(1)}\right)^{v-\eta}\right)+\ldots+\operatorname{dim}\left(\left({ }_{s} M_{(s)} / s-1 M_{(s)}\right)^{v-\eta}\right)$, where each $\left({ }_{i} M_{(1)} /{ }_{i-1} M_{(1)}\right)$ is a highest weight module of highest weight $v^{\prime}<v$, hence $v-\eta=v^{\prime}-\eta^{\prime}, \eta^{\prime}<\eta$. Equation (17) now follows from the induction hypothesis;

2) $\operatorname{dim} M^{v}=2$. This case is similar but we need to apply the argument of 1) twice. Finally, for general $M \in \mathscr{C}$, we apply Lemma 5.3 and the proof for highest weight modules. Q.E.D.

Remark. Proposition 5.4 implies that the numbers $(M: L(V(\mu))$ are independent of the choices apparently made in their definition.

We denote by $\aleph(\mathscr{C})$ the set of all characters of elements of $\mathscr{C}$. Proposition 5.5. Let $\mathbf{a} \in \mathfrak{\aleph}(\mathscr{C})$. If $\mathbf{a}=\sum_{\mu} a_{\mu} \operatorname{ch} L(V(\mu))=\sum_{\mu} b_{\mu} \operatorname{ch} L(V(\mu))$, then
$a_{\mu}=b_{\mu}$ for all $\mu \in \mathfrak{h}^{*}$.

Proof. Let $\mu_{1}$ be maximal so that $a_{\mu} \neq 0$. Then $a_{\mu_{1}} \operatorname{dim} L\left(V\left(\mu_{1}\right)\right)^{\mu_{1}} q^{\mu_{1}}$ $=b_{\mu_{1}} \operatorname{dim} L\left(V\left(\mu_{1}\right)\right)^{\mu_{1}} q^{\mu_{1}}$. Hence $a_{\mu_{1}}=b_{\mu_{2}}$. Suppose we have showed that $a_{\mu}=b_{\mu}$ for all $\mu$ such that $\mu_{1}-\mu<\eta \in Q^{+} \cup\{0\}$, and let $\mu$ be so that $\mu_{1}-\mu=\eta \in Q^{+} \cup\{0\}$. Then

$$
\sum_{\mu \leqq \mu_{1}+\eta} a_{\mu} \operatorname{ch} L(V(\mu))=\sum_{\mu \leqq \mu_{1}+\eta} b_{\mu} \operatorname{ch} L(V(\mu)) .
$$

Then $\quad a_{\mu_{1}+\eta} \operatorname{dim} L\left(V\left(\mu_{1}+\eta\right)\right)^{\mu_{1}+\eta} q^{\mu_{1}+\eta}=b_{\mu_{1}+\eta} \operatorname{dim} L\left(V\left(\mu_{1}+\eta\right)\right)^{\mu_{1}+\eta} q^{\mu_{1}+\eta}$, i.e. $a_{\mu_{1}+\eta}=b_{\mu_{1}+\eta}$. The result now follows by induction. Q.E.D.

Proposition 5.6. Let $M \in \mathscr{C}$. Then $L(V(\mu))$ is a subquotient of $M$ if and only if $(M: L(V(\omega)) \neq 0$.

Proof. If $(M: L(V(\mu)) \neq 0$, then $L(V(\mu))$ is a subquotient of $M$, by the definition of $(M: L(V(\mu))$. Conversely, let $M \supset N \supset P, N, P \in \mathscr{C}$ such that $N / P \simeq L(V(\mu))$. Then $\operatorname{ch} N=\operatorname{ch} P+\operatorname{ch} L(V(\mu))$. Also $\operatorname{ch} M=\operatorname{ch} N+\operatorname{ch} M / N$. By Proposition 5.4 $(M: L(V(\mu)) \geqq 1$. Q.E.D.

If $\mathbf{a}=\operatorname{ch} M \in \mathfrak{\aleph}(\mathscr{C})$ we define $(\mathbf{a}: \operatorname{ch} L(V(\mu))$ as $(M: L(V(\mu))$.

Proof of Theorem 5.1. Let $L(V(\mu))$ be a subquotient of $M(V(\lambda))$. We saw earlier that the maximal proper submodule of $M(V(\lambda))$ is $M(V(\lambda))_{(1)}$. Therefore, $\mu=\lambda$ or $L(V(\mu))$ is a subquotient of $M(V(\lambda))_{(1)}$. By Proposition 5.6, either $\mu=\lambda$ or $\left(M(V(\lambda))_{(1)}: L(V(\mu))\right) \neq 0$. By Proposition 5.2, $\mu=\lambda$ or $\sum_{i \in \frac{1}{2} \mathbb{N}_{+}(\lambda)} a(i)(\operatorname{ch} M(V(\lambda-i \delta)): \operatorname{ch} L(V(\mu))) \neq 0$. Hence, $\mu=\lambda$ or $L(V(\mu))$ is a subquotient of $M(V(\lambda-i \delta))$ for some $i \in \frac{1}{2} \mathbb{N}_{+}(\lambda)$, by Proposition 5.6. By induction, we obtain $\mu \uparrow \lambda$. Q.E.D. 


\section{Appendix. Central Extensions of $\mathbb{Z}_{\mathbf{2}}$-Graded Lie Algebras}

In this appendix we extend some definitions and classic results on central extensions of Lie algebras to $\mathbb{Z}_{2}$-graded Lie algebras.

As in Sect. 2, all homomorphisms of $\mathbb{Z}_{2}$-graded Lie algebras preserve the $\mathbb{Z}_{2}$-grading. Furthermore, all short exact sequences

$$
0 \rightarrow \mathfrak{b} \stackrel{\iota}{\longrightarrow} \mathfrak{e} \stackrel{\pi}{\longrightarrow} \mathfrak{a} \rightarrow 0
$$

of $\mathbb{Z}_{2}$-graded Lie algebras are $\mathbb{Z}_{2}$-split, i.e., are such that $\pi$ has a linear right inverse that preserves the $\mathbb{Z}_{2}$-grading (or equivalently, $\imath$ has a linear left inverse that preserves the $\mathbb{Z}_{2}$-grading). (1) is called an extension of $\mathfrak{a}$ by $\mathfrak{b}$. A morphism of (1) to another extension

$$
0 \rightarrow \mathfrak{b}^{\prime} \stackrel{\iota^{\prime}}{\longrightarrow} \mathfrak{e}^{\prime} \stackrel{\pi^{\prime}}{\longrightarrow} \mathfrak{a}^{\prime} \rightarrow 0
$$

is a triplet $(\psi, \phi, \varrho)$ of homomorphisms: $\psi: \mathfrak{b} \rightarrow \mathfrak{b}^{\prime}, \phi: \mathfrak{e} \rightarrow \mathfrak{e}^{\prime}$, and $\varrho: \mathfrak{a} \rightarrow \mathfrak{a}^{\prime}$ such that $\phi \circ l=l^{\prime} \circ \psi$ and $\pi^{\prime} \circ \phi=\varrho \circ \pi$.

For any $\mathbb{Z}_{2}$-graded Lie algebra $a$ we denote by $z(\mathfrak{a})$ the center of $\mathfrak{a}$, i.e., $z(\mathfrak{a})=\{X \in \mathfrak{a} \mid[x, \mathfrak{a}]=0\}$. If $\mathfrak{b} \leqq z(\mathfrak{e})$ in (1), then (1) is said to be a central extension of $\mathfrak{a}$. A morphism of the central extension (1) to a central extension

$$
0 \rightarrow \mathfrak{b}^{\prime} \stackrel{\mathfrak{t}^{\prime}}{\longrightarrow} \mathfrak{e}^{\prime^{\pi^{\prime}}} \stackrel{\longrightarrow}{\longrightarrow} \rightarrow 0
$$

is a morphism $(\psi, \phi, \varrho)$ of extensions from (1) to (3) such that $\varrho=\left.\mathrm{id}\right|_{\alpha}$. (Here, id $\left.\right|_{\alpha}$ denotes the identity homomorphism of $\mathfrak{a}$, for any $\mathbb{Z}_{2}$-graded Lie algebra $\mathfrak{a}$.) The extension (1) is said to be equivalent to

$$
0 \rightarrow \mathfrak{b} \stackrel{\iota^{\prime}}{\longrightarrow} \mathfrak{e}^{\prime} \stackrel{\pi^{\prime}}{\longrightarrow} \mathfrak{a} \rightarrow 0
$$

if there is a morphism $(\psi, \phi, \varrho)$ of extensions from (1) to (4) such that $\psi=\left.\mathrm{id}\right|_{\mathfrak{b}}$ and $\varrho=\left.\mathrm{id}\right|_{\mathrm{a}} . \phi$ is necessarily an isomorphism, i.e., $\phi$ has a two-sided inverse homomorphism which preserves the $\mathbb{Z}_{2}$-grading. Therefore, equivalence of extensions of $\mathfrak{a}$ by $\mathfrak{b}$ is an equivalence relation.

Let $V$ be a $\mathbb{Z}_{2}$-graded vector space: $V=V_{\overline{0}} \oplus V_{\overline{1}}$. A bilinear map $\alpha: \mathfrak{a} \times \mathfrak{a} \rightarrow V$ will always mean one that preserves the $\mathbb{Z}_{2}$-grading, in the sense that $\alpha\left(\mathfrak{a}_{\gamma_{1}} \times \mathfrak{a}_{\gamma_{2}}\right)$ $C V_{\gamma_{1}+\gamma_{2}}, \gamma_{1}, \gamma_{2} \in \mathbb{Z}_{2}$. A bilinear map $\alpha: \mathfrak{a} \times \mathfrak{a} \rightarrow V$ is skew-symmetric if

$$
\alpha(X, Y)=-(-1)^{\gamma_{1} \gamma_{2}} \alpha(Y, X), X \in \mathfrak{a}_{\gamma_{1}}, Y \in \mathfrak{a}_{\gamma_{2}}, \gamma_{1}, \gamma_{2} \in \mathbb{Z}_{2} .
$$

We denote by $Z^{2}(\mathfrak{a}, V)$ the space of all skew-symmetric bilinear maps $\alpha: \mathfrak{a} \times \mathfrak{a} \rightarrow V$ such that the following condition holds:

$$
(-1)^{\gamma_{1} \gamma_{3}} \alpha(X,[Y, Z])+(-1)^{\gamma_{2} \gamma_{1}} \alpha(Y,[Z, X])+(-1)^{\gamma_{3} \gamma_{2}} \alpha(Z,[X, Y])=0,
$$

where $X \in \mathfrak{a}_{\gamma_{1}}, Y \in \mathfrak{a}_{\gamma_{2}}, Z \in \mathfrak{a}_{\gamma_{3}}, \gamma_{1}, \gamma_{2}, \gamma_{3} \in \mathbb{Z}_{2}$. The elements of $Z^{2}(a, V)$ are called cocycles and (6) is the cocycle condition for $\mathbb{Z}_{2}$-graded Lie algebras. Let $B^{2}(\mathfrak{a}, V)$ be the space of all skew-symmetric bilinear maps $\alpha: \mathfrak{a} \times \mathfrak{a} \rightarrow V$ such that 
$\alpha(X, Y)=g([X, Y])$ for all $X, Y \in \mathfrak{a}$ and some linear map $g: \mathfrak{a} \rightarrow V$ such that $g\left(\mathfrak{a}_{\gamma}\right)$ $\subset V_{\gamma}$, for all $\gamma \in \mathbb{Z}_{2}$. The elements of $B^{2}(\mathfrak{a}, V)$ are the coboundaries. We note that condition $(L 2)$ of Sect. 2 implies that $B^{2}(\mathfrak{a}, V) \subset Z^{2}(\mathfrak{a}, V)$. Set $H^{2}(\mathfrak{a}, V)$ $=Z^{2}(\mathfrak{a}, V) / B^{2}(\mathfrak{a}, V) . H^{2}(\mathfrak{a}, V)$ is the second cohomology group of a with coefficients in $V$.

Next, we show how to associate to each element $\alpha$ in $Z^{2}(\mathfrak{a}, \mathfrak{b}), \mathfrak{b}$ abelian, $a$ central extension of $\mathfrak{a}$ by $\mathfrak{b}$. Let $\mathfrak{a}_{\alpha}$ denote the $\mathbb{Z}_{2}$-graded vector space $\mathfrak{a} \oplus \mathfrak{b}$, where $(\mathfrak{a} \oplus b)_{\gamma}=\mathfrak{a}_{\gamma} \oplus \mathfrak{b}_{\gamma}, \gamma \in \mathbb{Z}_{2}$. For $(X, v),(Y, w) \in\left(\mathfrak{a}_{\alpha}\right)_{\gamma}$, set

$$
[(X, v),(Y, w)]_{\alpha}=([X, Y], \alpha[X, Y]) .
$$

Clearly, $[,]_{\alpha}$ extends to a bilinear map from $\mathfrak{a}_{\alpha} \times \mathfrak{a}_{\alpha}$ to $\mathfrak{a}_{\alpha}$ and $\left[\left(\mathfrak{a}_{\alpha}\right)_{\gamma_{1}},\left(\mathfrak{a}_{\alpha}\right)_{\gamma_{2}}\right]$ $C\left(\mathfrak{a}_{\alpha}\right)_{\gamma_{1}+\gamma_{2}}, \gamma_{1}, \gamma_{2} \in \mathbb{Z}_{2}$. Condition (L1) of Sect. 2 follows from (5) and condition ( $\left.L 2\right)$ of Sect. 2 follows from (6), so that $\left(\mathfrak{a}_{\alpha},[,]_{\alpha}\right)$ is a $\mathbb{Z}_{2}$-graded Lie algebra. We associate to $\alpha \in Z^{2}(\mathfrak{a}, \mathfrak{b})$ the central extension

$$
0 \rightarrow \mathfrak{b} \stackrel{\imath_{\alpha}}{\longrightarrow} \mathfrak{a}_{\alpha} \stackrel{\pi_{\alpha}}{\longrightarrow} \mathfrak{a} \rightarrow 0
$$

where $t_{\alpha}$ is the obvious inclusion and $\pi_{\alpha}$ is the projection onto a. Furthermore, let $\alpha, \alpha^{\prime} \in Z^{2}(\mathfrak{a}, \mathfrak{b})$ and $\alpha^{\prime}-\alpha \in B^{2}(\mathfrak{a}, \mathfrak{b})$, i.e., there is $\mathfrak{g}: \mathfrak{a} \rightarrow \mathfrak{b}$ linear, preserving the $\mathbb{Z}_{2}$-grading, such that $\left(\alpha^{\prime}-\alpha\right)(X, Y)=g([X, Y])$, for all $X, Y \in \mathfrak{a}$. If we define $\phi:\left(\mathfrak{a}_{\alpha}\right)_{\gamma} \rightarrow\left(\mathfrak{a}_{\alpha^{\prime}}\right)_{\gamma}$ by $\phi((X, v))=(X, v+g(X)), X \in \mathfrak{a}_{\gamma}, v \in \mathfrak{b}_{\gamma}$, then $\left(\left.\mathrm{id}\right|_{\mathfrak{b}}, \phi,\left.\mathrm{id}\right|_{\mathfrak{a}}\right)$ is a morphism from (8) to

$$
0 \rightarrow \mathfrak{b} \stackrel{l_{\alpha^{\prime}}}{\longrightarrow} \mathfrak{a}_{\alpha^{\prime}} \stackrel{\pi_{\alpha^{\prime}}}{\longrightarrow} \mathfrak{a} \rightarrow 0
$$

Conversely, if (1) is a given central extension of $\mathfrak{a}$, i.e., $\mathfrak{b} \in \mathfrak{z}_{\mathfrak{e}}$, let $\beta: \mathfrak{a} \rightarrow \mathfrak{e}$ be a linear map which preserves the $\mathbb{Z}_{2}$-grading and is such that $\pi \circ \beta=\left.\mathrm{id}\right|_{\mathbf{a}}$. Set $\alpha_{0}(X, Y)$ $=[\beta(X), \beta(Y)]-\beta([X, Y]), X, Y \in \mathfrak{a}$. Clearly, $\pi\left(\alpha_{0}(X, Y)\right)=0$, so that $\alpha_{0}=1 \circ \alpha$, where $\alpha$ is a skew-symmetric, bilinear map from $\mathfrak{a} \times \mathfrak{a}$ to $\mathfrak{b}$. Now, let $X \in \mathfrak{a}_{\gamma_{1}}, Y \in \mathfrak{a}_{\gamma_{2}}$, $Z \in \mathfrak{a}_{\gamma_{3}}$. Then

$$
\begin{aligned}
&(-1)^{\gamma_{1} \gamma_{3}} \alpha_{0}(X,[Y, Z])+(-1)^{\gamma_{2} \gamma_{1}} \alpha_{0}(Y,[Z, X])+(-1)^{\gamma_{3} \gamma_{2}} \alpha_{0}(Z,[X, Y]) \\
&=(-1)^{\gamma_{1} \gamma_{3}}([\beta(X), \beta([Y, Z])]-\beta([X,[Y, Z]])) \\
&+(-1)^{\gamma_{2} \gamma_{1}}([\beta(Y), \beta([Z, X])]-\beta([Y,[Z, X]])) \\
&+(-1)^{\gamma_{3} \gamma_{2}}([\beta(Z), \beta([X, Y])]-\beta([Z,[X, Y]])) \\
&=(-1)^{\gamma_{1} \gamma_{3}}([\beta(X), \beta([Y, Z])]-[\beta(X),[\beta(Y), \beta(Z)]]) \\
&+(-1)^{\gamma_{2} \gamma_{1}}([\beta(Y), \beta([Z, X])]-[\beta(Y),[\beta(Z), \beta(X)]]) \\
&+(-1)^{\gamma_{3} \gamma_{2}}([\beta(Z), \beta([X, Y])]-[\beta(Z),[\beta(X), \beta(Y)]]) \\
&=(-1)^{\gamma_{1} \gamma_{3}}[\beta(X), \beta([Y, Z])-[\beta(Y), \beta(Z)]] \\
&+(-1)^{\gamma_{2} \gamma_{1}}[\beta(Y), \beta([Z, X])-[\beta(Z), \beta(X)]] \\
&+(-1)^{\gamma_{3} \gamma_{2}}[\beta(Z), \beta([X, Y])-[\beta(X), \beta(Y)]] \\
&=(-1)^{\gamma_{1} \gamma_{3}}\left[\beta(X),-\alpha_{0}(Y, Z)\right]+(-1)^{\gamma_{2} \gamma_{1}}\left[\beta(Y),-\alpha_{0}(Z, X)\right] \\
&+(-1)^{\gamma_{3} \gamma_{2}}\left[\beta(Z),-\alpha_{0}(X, Y)\right]=0,
\end{aligned}
$$


by Sect. $2(L 2)$ and since $(\imath \circ \alpha)(\mathfrak{a} \times \mathfrak{a}) \subset l(\mathfrak{b}) \subset \mathfrak{z}_{\mathrm{e}}$. Therefore, $\alpha \in Z^{2}(\mathfrak{a}, \mathfrak{b})$ and we have associated to each central extension of $\mathfrak{a}$ by $\mathfrak{b}$, an element of $Z^{2}(\mathfrak{a}, \mathfrak{b})$. It is immediate to check that the equivalence class of the latter in $H^{2}(\mathfrak{a}, \mathfrak{b})$ does not depend on the choice of the map $\beta$ used above. Now, suppose that there is a morphism $\left(\left.\mathrm{id}\right|_{\mathfrak{b}}, \phi,\left.\mathrm{id}\right|_{a}\right)$ from the central extension (1) to the central extension (4). Let $\beta$ be a right inverse of $\pi$ that preserves the $\mathbb{Z}_{2}$-grading, as before. Let $\alpha$ be the element of $Z^{2}(\mathfrak{a}, \mathfrak{b})$ corresponding to (1) and constructed via $\beta$. Since $\phi \circ \beta$ is a right inverse of $\pi$ that preserves the $\mathbb{Z}_{2}$-grading, it is easy to see that (4) corresponds to the equivalence class of $\alpha$ in $H^{2}(a, b)$, by the previous observation. We have, thus, associated to each element $[\alpha]$ of $H^{2}(\mathfrak{a}, \mathfrak{b})$, an equivalence class $\Phi[\alpha]$ of central extensions of $\mathfrak{a}$ by $\mathfrak{b}$, and, to each equivalence class $[(l, \pi)]$ of central extensions of $\mathfrak{a}$ by $\mathfrak{b}$, an element $\Psi([(l, \pi)])$ of $H^{2}(\mathfrak{a}, \mathfrak{b})$. Here $(l, \pi)$ denotes the central extension (1). Let $C E(\mathfrak{a}, \mathfrak{b})$ denote the set of all equivalence classes of central extensions of $\mathfrak{a}$ by $\mathfrak{b}$.

Theorem 1. The map $\Phi: H^{2}(\mathfrak{a}, \mathfrak{b}) \rightarrow C E(\mathfrak{a}, b)$ is a bijection with inverse $\Psi: C E(\mathfrak{a}, b)$ $\rightarrow H^{2}(\mathfrak{a}, \mathfrak{b})$.

Proof. Let $(l, \pi)$ denote the element (1) of $C E(\mathfrak{a}, \mathfrak{b})$. Let $\Psi([(l, \pi)])=[\alpha]$ as constructed above. Let $\left(i_{\alpha}, \pi_{\alpha}\right)$ be the central extension (8). It is easy to see that $\phi: \mathfrak{a}_{\alpha}$ $\rightarrow \mathfrak{e}$ defined by $\phi((X, v))=\beta(X)+\imath(v),(X, v) \in \mathfrak{a}_{\alpha}$, where $\beta$ is the right $\left(\mathbb{Z}_{2}\right.$-graded $)$ inverse of $\pi$, is a morphism from $\left(l_{\alpha}, \pi_{\alpha}\right)$ to $(l, \pi)$, so that $\Phi \circ \Psi$ is the identity map on $C E(\mathfrak{a}, \mathfrak{b})$. It is immediate from the above constructions that $\Psi \circ \Phi$ is the identity on $H^{2}(\mathfrak{a}, \mathfrak{b})$. Q.E.D.

As in Sect. 2, we consider, for any $\mathbb{Z}_{2}$-graded vector space $V$, the tensor algebra $T(V)=\bigoplus_{p=0}^{\infty} T^{P}(V)$. Let $K$ be the two-sided $\left(\mathbb{Z}_{2}\right.$-graded $)$ ideal in $T(V)$ generated by the elements of the form $X \otimes Y+(-1)^{\gamma_{1} \gamma_{2}} Y \otimes X, X \in V_{\gamma_{1}}, Y \in V_{\gamma_{2}}, \gamma_{1}, \gamma_{2} \in \mathbb{Z}_{2}$. The exterior algebra of $V$ is $\Lambda(V)=T(V) / K=\bigoplus_{p=0}^{\infty} \Lambda^{P}(V)$, where $\Lambda^{P}(V)$ is the image of $T^{P}(V)$ in $\Lambda(V)$ by the quotient map $\delta: T(V) \rightarrow \Lambda(V)$.

For any $\mathbb{Z}_{2}$-graded Lie algebra a, let $L$ be the subspace of $\Lambda^{2}(\mathfrak{a})$ generated by the elements of the form

$$
(-1)^{\gamma_{1} \gamma_{3}} X \wedge[Y, Z]+(-1)^{\gamma_{2} \gamma_{1}} Y \wedge[Z, X]+(-1)^{\gamma_{3} \gamma_{2}} Z \wedge[X, Y] .
$$

The second homology group of $\mathfrak{a}$ with trivial coefficients is $H_{2}(\mathfrak{a}, \mathbb{C})=\Lambda^{2}(\mathfrak{a}) / L$. Following [10, Lemma I.10], we set $\alpha(X, Y)=\delta(X \wedge Y)$. By (9) one sees that $\alpha \in Z^{2}\left(\mathfrak{a}, H_{2}(\mathfrak{a}, \mathbb{C})\right)$. Let $\Phi([\alpha])$ be the central extension

$$
0 \rightarrow H_{2}(\mathfrak{a}, \mathbb{C}) \stackrel{l_{\alpha}}{\longrightarrow} \mathfrak{a}_{\alpha} \stackrel{\pi_{\alpha}}{\longrightarrow} \mathfrak{a} \rightarrow 0,
$$

as constructed prior to Theorem 1 .

A central extension (1) of $\mathfrak{a}$ is said to be universal if, given any central extension (3) of $\mathfrak{a}$, there is a unique morphism from the central extension (1) to the central extension (3). Proceeding as in the proof of Lemma I.10 of [10] we obtain the $\mathbb{Z}_{2}$-graded analog of that lemma.

Proposition 2. If $[\mathfrak{a}, \mathfrak{a}]=\mathfrak{a}$ then the central extension (10) is universal. 
Acknowledgements. A. Meurman would like to thank I. Frenkel for stimulating discussions about vertex operators and the Virasoro algebra. A. Rocha gratefully acknowledges D. Friedan for pointing out the role played by the Neveu-Schwarz and Ramond algebras in 2-dimensional field theories and, in particular, in string theory.

\section{References}

1. Corwin, L., Ne'eman, Y., Sternberg, S.: Graded Lie algebras in mathematics and physics (Bose-Fermi symmetry). Rev. Mod. Phys. 47, 573-603 (1975)

2. Feigin, B.L., Fuchs, D.B.: Funkts. Anal. Prilozh. 16, 47-63 (1982)

3. Feingold, A., Frenkel, I.B.: Classical affine algebras. Adv. Math. (to appear)

4. Frenkel, I.B.: Spinor representations of affine Lie algebras. Proc. Natl. Acad. Sci. USA 77, 6303-6306 (1980)

5. Frenkel, I.B.: Private communication to A. Meurman

6. Frenkel, I.B., Kac, V.G.: Invent. Math. 62, 23-66 (1980)

7. Friedan, D.: Private communication to A. Rocha-Caridi

8. Friedan, D., Qiu, Z., Shenker, S.H.: Superconformal invariance in two dimensions and the tricritical Ising model. Phys. Lett. 151 B, 37 (1985)

9. Garland, H., Lepowsky, J.: Invent. Math. 34, 37-76 (1976)

10. Garland, H.: Publ. Math. Inst. Hautes Etud. Sci. 52, (1980)

11. Goodman, R., Wallach, N.R.: Projective Unitary Positive-Energy Representations of $\operatorname{Diff}\left(S^{1}\right)$, preprint (1984)

12. Goodman, R., Wallach, N.R.: J. Reine Angew. Math. 347, 69-133 (1984)

13. Kac, V.G.: Proceedings of the International Congress of Mathematicians, Helsinki (1978)

14. Kac, V.G.: Group theoretical methods in physics. In: Lecture Notes in Physics. Beiglböck, W., Böhm, A., Takasugi, E. (eds.), Vol. 94, p. 441. Berlin, Heidelberg, New York: Springer 1979

15. Kac, V.G.: Adv. Math. 26, 8-96 (1977)

16. Kac, V.G.: Differential geometrical methods in mathematical physics II. Lecture Notes in Mathematics. Bleuler, K., Petri, H., Reetz, A. (eds.), Vol. 676, pp. 597-626. Berlin, Heidelberg, New York: Springer 1978

17. Kac, V.G., Peterson, D.: Spin and wedge representations of infinite-dimensional Lie algebras and groups. Proc. Nat1. Acad. Sci. USA 78, 3308-3312 (1981)

18. Milnor, J., Moore, J.: Ann. Math. 81, 211-264 (1965)

19. Neveu, P., Schwarz, J.H.: Factorizable dual model of pions. Nucl. Phys. B 31, 86-112 (1971)

20. Ramond, P.: Dual theory for free fermions. Phys. Rev. D 3, 2415-2418 (1971)

21. Retakh, V.S., Feigin, B.L., Usp. Mat. Nauk. 37, 233-234 (1982)

22. Rocha-Caridi, A.: Vertex operators in mathematics and physics. Proceedings of a Conference, November 10-17, 1983. In: Mathematical Sciences Research Institute Publications. Lepowsky, J., Mandelstam, S., Singer, I.M. (eds.), Vol. 3. Berlin, Heidelberg, New York: SpringerVerlag 1984

23. Rocha-Caridi, A., Wallach, N.R.: Invent. Math. 72, 57-75 (1983)

24. Rocha-Caridi, A., Wallach, N.R.: Math. Z. 180, 151-177 (1982)

25. Segal, G.: Unitary representations of some infinite dimensional groups. Commun. Math. Phys. 80, 301-342 (1981)

26. Shapovalov, N.N.: Funct. Anal. Appl. 6, 307-312 (1972)

27. Thorn, C.B.: Computing the Kac determinant using dual model techniques and more about the no-ghost theorem. Nucl. Phys. B 248, 551 (1984)

Communicated by S.-T. Yau

Received June 3, 1985 